\title{
Event-Based Control of Depth of Hypnosis in Anesthesia
}

\author{
Luca Merigo $^{1} \quad$ Manuel Beschi ${ }^{2} \quad$ Fabrizio Padula ${ }^{3}$ \\ Nicola Latronico ${ }^{4}$ Massimiliano Paltenghi $^{5} \quad$ Antonio Visioli ${ }^{6}$ \\ ${ }^{1}$ Dipartimento di Ingegneria dell'Informazione \\ University of Brescia, Italy \\ e-mail: l.merigo001@unibs.it \\ ${ }^{2}$ Istituto di Tecnologie Industriali e Automazione \\ National Research Council Milan, Italy \\ e-mail: manuel.beschi@itia.cnr.it \\ ${ }^{3}$ Department of Mathematics and Statistics \\ Curtin University, Australia \\ e-mail: fabrizio.padula@curtin.edu.au \\ ${ }^{4}$ Department of Surgery, Radiology, and Public Health, \\ University of Brescia, Italy \\ e-mail: nicola.latronico@unibs.it \\ ${ }^{5}$ Spedali Civili di Brescia, Brescia, Italy \\ e-mail: maxpaltenghi@gmail.com \\ ${ }^{6}$ Dipartimento di Ingegneria Meccanica e Industriale \\ University of Brescia, Italy \\ e-mail: antonio.visioli@unibs.it
}

\begin{abstract}
Background and Objective: In this paper we propose the use of an event-based control strategy for the closed-loop control of the depth of hypnosis in anesthesia by using propofol administration and the bispectral index as a controlled variable.

Methods: A new event generator with high noise-filtering properties is employed in addition to a PIDPlus controller. The tuning of the parameters is performed off-line by using genetic algorithms by considering a given data set of patients.

Results: The effectiveness and robustness of the method is verified in simulation by implementing a Monte Carlo method to address the intra-patient and inter-patient variability. A comparison with a standard PID control structure shows that the event-based control system achieves a reduction of the total variation of the manipulated variable of $93 \%$ in the induction phase and of $95 \%$ in the maintenance phase.

Conclusions: The use of event based automatic control in anesthesia yields a fast induction phase with bounded overshoot and an acceptable disturbance rejection. A comparison with a standard PID control structure shows that the technique effectively mimics the behavior of the anesthesiologist by providing a significant decrement of the total variation of the manipulated variable.
\end{abstract}

Keywords: Depth of hypnosis control, PID control, Event-based control, noise filtering. 


\section{List of abbreviations}

AEP: auditory evoked potential

BIS: bispectral index scale

BIS-NADIR: lowest value of the bispectral index scale

DoH: depth of hypnosis

EEG: electroencephalogram

IAE: integrated absolute error

MDPE: median performance error

MDAPE: median absolute performance error

PE: performance error

PID: proportional-integral-derivative

PD: pharmacodynamics

PK: pharmacokinetics

PSD: power spectral density

Q: quantity of administered drug

SOD: send-on-delta

ST10: $10 \%$ settling time

ST20: $20 \%$ settling time

TCI: target controlled infusion

TIVA: total intravenous anesthesia

TT: time to target

TV: total variation

US: undershoot 


\section{Introduction}

In modern medicine the control technology influences different clinical practices (consider, for example, robotic surgery or automatic implantable defibrillators [37]). In fact, new discoveries in biology and the improved understanding of biological functions have contributed to the development of new sensors, actuators and mathematical models of the human body response to administration of drugs. This has allowed the introduction of control systems also in drug administration contexts, among which the closed-loop control of anesthesia during surgery is one of the most important [8]. In particular, the administration of propofol in a total intravenous anesthesia (TIVA) can be automatically regulated using the bispectral index scale (BIS, AspectMedical Systems, Norwood, USA) as controlled variable in order to achieve a predefined level of depth of hypnosis (DoH). The BIS signal provides an estimation of the hypnosis level of the patient, based on the electroencephalogram (EEG) bispectral analysis, with a dimensionless number, which ranges from 0 (equivalent to EEG silence) to 100 [46]. Instrumentation for BIS measurement is widespread in operating rooms and it is widely accepted by clinical practitioners.

In the clinical practice the anesthesiologist manually regulates the drugs infusion and the correct propofol administration for a desired level of hypnosis is decided relying on experience, on recommended doses, and on the trends of some vital signs of the patient. The anesthesiologist also administers other necessary drugs for analgesia and neuromuscular blockade. Regarding $\mathrm{DoH}$, the clinical practice usually involves an initial induction phase where a propofol intravenous bolus is used to drive the patient from consciousness to the required hypnotic state in a short time. The rapidity of this phase may give undershoot of the BIS level over the predefined DoH target that might imply dangerous hypotension [23]. During surgery, the anesthesiologist has then to keep a specified BIS level (usually between 40 and 60) despite the occurrence of disturbances mainly related to noxious stimuli. In this maintenance phase, some other boluses could be necessary in emergency cases. Finally, there is the emergence phase where, by stopping the administration of the drugs, the patient recovers from the anesthesia.

A model of the human body drug response is necessary to develop an automatic control system that replicates and improves this clinical procedure. The patient model takes into account the relationship between the propofol infusion rate and the drug effect by means of pharmacokinetic (PK) and pharmacodynamic (PD) models [33]. Pharmacokinetics refers to the infusion, distribution and elimination of the drugs in the body, while pharmacodynamics describes the relationship between the blood concentration of the drug and its clinical effect. The pharmacodynamic model comprises a fictitious compartment, call effect-side, and a nonlinear function, called Hill function, that completes the model by expressing the relation between the 
effect-site drug concentration and the clinical effect, giving the BIS level. Based on this model, an open-loop system called target controlled infusion (TCI) has been developed and introduced in surgery for propofol automatic infusion [19]. The anesthesiologist selects the desired blood concentration or the effect site target concentration and the TCI calculates an appropriate bolus based on internal model inversion to achieve the selected value. The anesthesiologist then closes the loop manually to compensate for the modeling uncertainties and the inter-patient variability.

The development of closed-loop control systems for propofol infusion provides significant benefits such as the reduction of the anesthesiologist workload (even if he/she has to be present in any case for supervision and intervention in case of emergency), the avoidance of the problems due to distraction or fatigue, the increase of the safety for the patient thanks to the continuous patient monitoring and, finally, the possible lower administration of drug with a faster post-operative recovery and the reduction of side effects. It has to be taken into account that both too large and too small values of BIS have to be avoided. Indeed, awakenings due to a too small infusion rate should be avoided, but also overdosing might yield cognitive impairment and post-operative delirium [12, 23, 24].

From a control systems point of view, the control problem consists in achieving a fast set-point response with almost no undershoot in the induction phase and a fast disturbance rejection in the maintenance phase. The controller must be also robust to compensate for the different physical characteristics of the patients.

Despite these difficulties, in the literature it is possible to find many applications of a simple proportional-integral-derivative (PID) controller [28]. Different design approaches have been used to design the controller. For example, in [14] the PID has been tuned by a simulation on a patients database. Another approach has been implemented in [52]: the patient model is estimated during the manual initial bolus and the PID is tuned based on it. Other design approaches presented in [18] and [22] have exploited other feedback signals, such as the $\mathrm{WAV}_{\mathrm{CNS}}[59]$ and the auditory evoked potential (AEP). A simplified problem has been addressed in [27], where only the maintenance phase is performed in closed loop. On the contrary, in [26] the complete problem has been studied by considering also the automatic infusion of the analgesia drug. Other studies have been presented in $[2,4,45]$. In these works, the PID controller has been implemented and compared with TCI systems or manual infusion obtaining encouraging results.

Other control methods, such as internal model control [1], model predictive control $[20,58]$, fractional control $[14,32], \mu$-synthesis [18], fuzzy control [5, 21], neural network based control [17], and positive control [41] have been also proposed to control the DoH in the considered framework, and relevant results have been achieved. In any case it has been recently shown 
that it is difficult to improve the performance achieved by a properly tuned PID controller [43].

However, in addition to the above mentioned control requirements, it has to be taken also into account that the BIS signal is very noisy and this might cause a significant decrement of the overall performance. In order to address this issue, in [25] the controller feedback is considered only if the signal quality index is bigger than a given threshold. In [41] a median filter has been used to elaborate BIS signal and filter the noise. Finally, in [43] a low-pass filter is applied on the error variable to reduce the noise and a simple technique for the tuning of the time constant has been developed.

In any case, the methods proposed in the literature until now suffer from the following problems:

- the noise in the control variable is significant because its strong reduction would yield an unacceptable decrement of the performance;

- the provided control action might not be intuitive for the anesthesiologist, who operates in any case as supervisor and needs to fully understand the behavior of the controller.

In this paper we propose an event-based control strategy based on a PIDPlus controller, where the control action is updated only when an event occurs [10]. In fact, the rationale of an event-based system is to exploit the control resources only if it is really necessary, that is, when an event occurs [35]. In this context, different event generator algorithms have been proposed in the literature [47]. The PIDPlus controller has already been applied successfully in wireless process control systems in order to reduce the transmissions from the sensor to the controller $[9,11,53]$. In the process control applications where it has been proposed so far, the PIDPlus controller exploits always the Send-On-Delta (SOD) sampling [34], which generates an event when the process variable changes more than a given threshold. However, in the DoH control, because of the strong nonlinearity of the system, the use of the standard SOD strategy might result in the sticking phenomenon [35], which eventually yields very long settling times.

In this paper we propose a new event generator algorithm with strong filtering capabilities to solve the problem of the noisy BIS signal without any significant decrement in the performance. In particular, the use of a suitable integral event condition together with a sampling criterion that averages the BIS signal between two consecutive events allows the elimination of the residual noise on the control variable, with less (useless) variations of the infusion rate.

Thus, the devised method presents the following advantages with respect to the other strategies proposed in the literature:

- it significantly reduces the effects of the measurement noise without a significant decrement of the performance; 
- it mimics the way of operating of the anesthesiologist as the behavior of the feedback controller is very close to the manual regulation of the drug infusion provided by the anesthesiologists, where the infusion rate is changed only if an event is detected;

- from the medical staff point of view, the behavior of the controller is clearly understandable, so that the anesthesiologist can easily monitor the system and perform emergency actions;

- a less stressful pump actuation is obtained thanks to the reduced number of changes in the control action.

Note that event-based controllers have never been applied to the control of anesthesia so far.

The main challenge in the design of an event-based PID controller is having more parameters to tune than a time-driven PID controller and this has been addressed by applying a genetic algorithm (where the induction and maintenance phases have been considered separately) to a benchmark set of 13 patients that are representative of a wide population [20, 38, 54]. A set of optimal tuning parameters to be used with any patient has been obtained. The robustness of the proposed control system with the obtained parameters has then been validated with a Monte Carlo method with respect to the inter- and intra-patient variability.

The paper is organized as follows. In Section 2 the pharmacokinetic and pharmacodynamic models of propofol are briefly reviewed and the benchmark set of patients is reported for the reader's convenience. The standard PIDPlus event-based controller is reviewed in Section 3 in order to highlight the main features. The proposed event-based controller is described in Section 4. Simulation results (including a comparison with the standard PID controller are presented in Section 5 and discussed in Section 6. Finally, conclusions are drawn in Section 7.

\section{Pharmacokinetic-pharmacodynamic modeling of propofol}

A PK-PD model describes the relationship between propofol infusion rate and the drug effect. Pharmacokinetics characterizes the relation between the infusion and the plasmatic concentration of the drug by describing the distribution and elimination of propofol in the body. The pharmacodynamics represents the relation between the plasmatic concentration of the drug and its effect site concentration. For propofol, the series of the PK-PD models can be described by a fourth-order system with two zeros whose parameters depend on the total body weight, height and gender of the patient (see $[49,50]$ for details). Note that the parameters are based on population 
averages, hence the real patient parameters are distributed according to the variances reported in [49].

Then, an additional static nonlinear function is added in series to PD to complete the propofol response model $[20,55,56]$. This sigmoid function, also known as Hill function, correlates the effect-site drug concentration and the clinical effect, represented by the bispectral index scale (BIS):

$$
B I S(t)=E_{0}-E_{\max }\left(\frac{C_{e}(t)^{\gamma}}{C_{e}(t)^{\gamma}+C_{e_{50}}^{\gamma}}\right)
$$

where $E_{0}$ is the baseline value representing the BIS level of the patient in the initial state before the infusion, $E_{\max }$ is the maximum reachable effect achieved by the infusion, $\gamma$ represents the steepness of the curve that represents the receptiveness of the patient to the drug and $C_{e_{50}}$ is the drug concentration necessary to reach the half maximal effect. The BIS is a dimensionless index derived from the EEG that represents the level of consciousness. A BIS value of 0 corresponds to EEG silence while a value of 100 denotes a fully conscious patient. Typically, a target BIS value during surgery is 50 but a value between 40 and 60 is sufficient to guarantee an adequate level of sedation $[3,8]$.

In order to select the parameters of the controller (see Section 4.2) and to evaluate its performance, the set of patient already used in $[20,38,54]$ has been considered. The values of the model parameters for the considered population are reported, for the reader's convenience in Table 1. Note that the dataset has been defined based on clinical studies in order to represent a wide range of people and can be therefore considered as a benchmark accepted by clinicians. In any case, the inter-patient and intra-patient variability is also evaluated by using a Monte Carlo method, see Section 5.3. The thirteenth individual of the considered population is a fictitious person whose parameters have been calculated as the algebraic mean of the other patients, and is therefore referred to as the average patient.

It is worth stressing that newborn and children are not taken into account in this paper as in clinical practice they are handled with different procedures because of their different PK-PD models $[13,31]$.

\section{PIDPlus control architecture}

In this section we briefly review the event-based control paradigm and the PIDPlus controller in order to make the proposed method and its application to the DoH control clearer.

Nowadays, thanks to the existence of a mature theory, the vast majority of control systems are based on periodical sampling of signals. Indeed, well established control design methods can be found in literature when signals are sampled at regular time intervals [16]. 


\begin{tabular}{|c|c|c|c|c|c|c|c|c|}
\hline Id & Age & $\mathrm{H}[\mathrm{cm}]$ & $\mathrm{W}[\mathrm{kg}]$ & Gender & $C_{e_{50}}$ & $\gamma$ & $E_{0}$ & $E_{\max }$ \\
\hline 1 & 40 & 163 & 54 & $\mathrm{~F}$ & 6.33 & 2.24 & 98.8 & 94.10 \\
\hline 2 & 36 & 163 & 50 & $\mathrm{~F}$ & 6.76 & 4.29 & 98.6 & 86.00 \\
\hline 3 & 28 & 164 & 52 & $\mathrm{~F}$ & 8.44 & 4.10 & 91.2 & 80.70 \\
\hline 4 & 50 & 163 & 83 & $\mathrm{~F}$ & 6.44 & 2.18 & 95.9 & 102.00 \\
\hline 5 & 28 & 164 & 60 & $\mathrm{M}$ & 4.93 & 2.46 & 94.7 & 85.30 \\
\hline 6 & 43 & 163 & 59 & $\mathrm{~F}$ & 12.00 & 2.42 & 90.2 & 147.00 \\
\hline 7 & 37 & 187 & 75 & $\mathrm{M}$ & 8.02 & 2.10 & 92.0 & 104.00 \\
\hline 8 & 38 & 174 & 80 & $\mathrm{~F}$ & 6.56 & 4.12 & 95.5 & 76.40 \\
\hline 9 & 41 & 170 & 70 & $\mathrm{~F}$ & 6.15 & 6.89 & 89.2 & 63.80 \\
\hline 10 & 37 & 167 & 58 & $\mathrm{~F}$ & 13.70 & 1.65 & 83.1 & 151.00 \\
\hline 11 & 42 & 179 & 78 & $\mathrm{M}$ & 4.82 & 1.85 & 91.8 & 77.90 \\
\hline 12 & 34 & 172 & 58 & $\mathrm{~F}$ & 4.95 & 1.84 & 96.2 & 90.80 \\
\hline 13 & 38 & 169 & 65 & $\mathrm{~F}$ & 7.42 & 3.00 & 93.1 & 96.58 \\
\hline
\end{tabular}

Table 1: Characteristic variables for the considered set of patients (H: heigth, W: weigth).

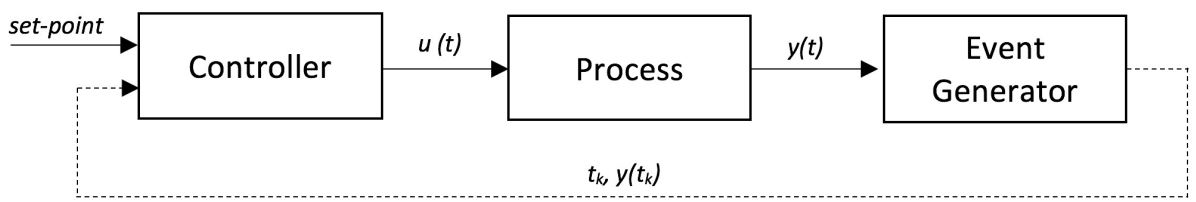

Figure 1: Block diagram of an event based control system.

However, recently, the event-based paradigm has been proposed as an effective solution to minimize the utilization of resources such as the energy and the network (this can be crucial, for example, in wireless or low power systems) [29]. In fact, an event-based system exploits the resources only if it is really necessary by sampling and transmitting signals only when an event occurs [35].

A block diagram of an event-based closed loop control system is shown in Figure 1. The system is composed by a process, an event generator and a controller. The event generator implements the event condition and, when the event condition becomes true, sends to the controller a sample of the continuous signal that receives as input.

An event occurs when a logical condition becomes true. The most typical event generator used in control systems is based on the so-called SendOn-Delta (SOD) sampling [34] (also called dead-band sampling, or leveltriggered sampling, or Lebesgue sampling [6]). This technique provides an 
event generation when a signal changes more than a given threshold $\Delta$. A synchronous condition that forces the triggering of the event is also commonly used because there are situations in which the logical condition can never be true [48]. With this condition an event is raised when the elapsed time without any triggering is higher than a predefined threshold $t_{\max }$. This is actually a safety condition that generates a set of periodical events especially during the steady state of the process. In particular, in DoH control, this condition can be considered as a safety element in the control of the DoH. In fact, especially during the maintenance phase, a set of periodical events is in any case generated due to the $t_{\max }$ condition (with possible small periodical changes in the infusion level).

In general, the SOD event condition on process output can be formalized as

$$
\left|g\left(y(t)-y\left(t_{\text {last }}\right)\right)\right| \geq \Delta \quad \text { OR } \quad t_{w} \geq t_{\max },
$$

where $g(\cdot)$ is a suitable mathematical function, $y\left(t_{\text {last }}\right)$ is the value of $y(t)$ at the previous event, $\Delta$ is the event threshold, and $t_{w}$ is the time elapsed without event triggering.

The controller receives as input the set-point value and the feedback action as in a standard feedback control scheme. The control action is however updated only when an event occurs, that is, when a new feedback value is available, and for this reason a specific controller is necessary to handle a non uniform sampling rate. In this context, one of the most relevant solution already applied in the process industry is the PIDPlus controller [30], which represents a modification of the standard PID controller in order to handle non-periodic measurements. The PIDPlus controller is based on the automatic reset implementation of a standard PID controller, as it is shown in Figure 2, where $K_{p}$ is the proportional gain, $T_{d}$ is the derivative time constant, $e$ is the control error, $u$ is the control variable, and the integral action is given by a positive feedback with a first-order filter whose time constant is the integral time constant $T_{i}$ and whose output is denoted as $f$. When an event occurs the new filter output is determined as

$$
f(t)=f\left(t_{\text {last }}\right)+\left(u\left(t_{\text {last }}\right)-f\left(t_{\text {last }}\right)\right)\left(1-e^{-\frac{\Delta t}{T_{i}}}\right),
$$

where $f\left(t_{\text {last }}\right)$ is the filter output for the last execution, $u\left(t_{\text {last }}\right)$ is the controller output for the last execution, and $\Delta t$ is the elapsed time between the current event and the previous one. The determined value $f(t)$ is used as the positive feedback signal. This particular controller implementation indeed maintains the last calculated filter output until a new event occurs. The derivative action, denoted as $u_{d}(t)$ is calculated only when an event occurs with the backward differences method. In order to avoid the well-known derivative kick phenomenon [7], it should be applied to the process variable, resulting in

$$
u_{d}(t)=T_{d} \frac{y(t)-y\left(t_{\text {last }}\right)}{t-t_{\text {last }}} .
$$




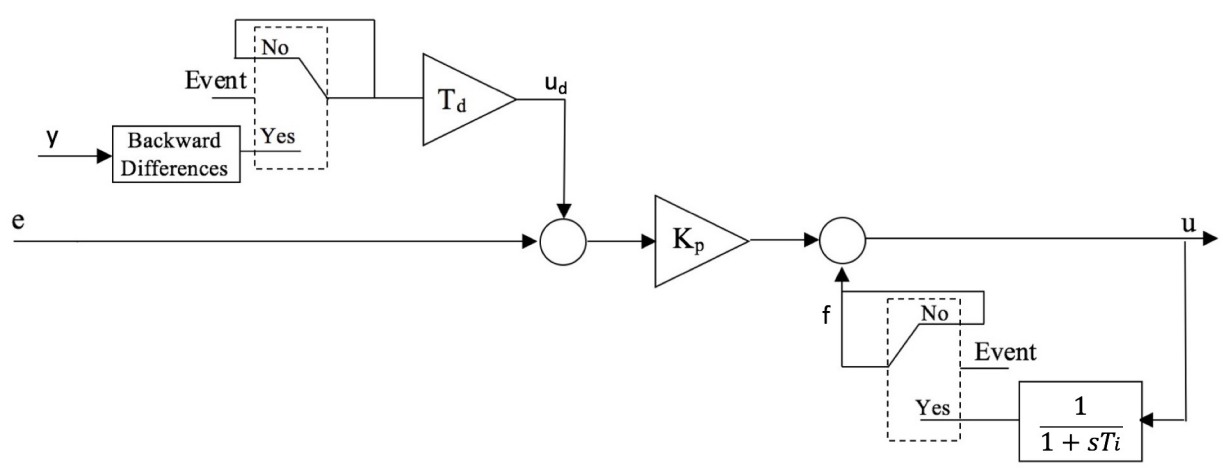

Figure 2: PIDPlus structure derived from automatic reset form of standard PID.

The derivative value is then maintained constant between two events.

It is worth noting that, despite its apparently complex formulation, the PIDPlus algorithm is exactly a standard discrete-time PID controller if events are evenly generated. Further, the event generator condition that has been used in the literature until now with a PIDPlus controller is the basic SOD condition

$$
\left|y(t)-y\left(t_{\text {last }}\right)\right| \geq \Delta \quad \text { OR } \quad t_{w} \geq t_{\max } .
$$

This event condition is unsuitable for noisy and nonlinear applications such as the DoH control because of the sticking phenomenon [35] and a new event condition will be therefore introduced in the next section in order to deal effectively with the high measurement noise of the BIS signal.

\section{Event-based control system design}

\subsection{Methodology}

In this paper a new event generator and the PIDPlus controller described in Section 3 will be used to design an event-based control system suitable for the model described in Section 2. In fact, in the propofol administration the use of resources is not an issue, but the event-based approach can be effectively used to filter the noise and to reproduce the typical clinical practice where the anesthesiologist regulates the drug infusion based on the events that occur during the surgery.

The aim is the automatic regulation of the propofol infusion rate to provide a transition of the patient DoH from the initial BIS level to the desired one and maintain the predefined BIS level during the maintenance phase. The BIS signal is therefore the feedback signal of the closed-loop system presented in Figure 3. In the block diagram, $r(t)$ is the set-point signal and $e(t)$ is the error variable. In clinical practice a typical reference value is 50 


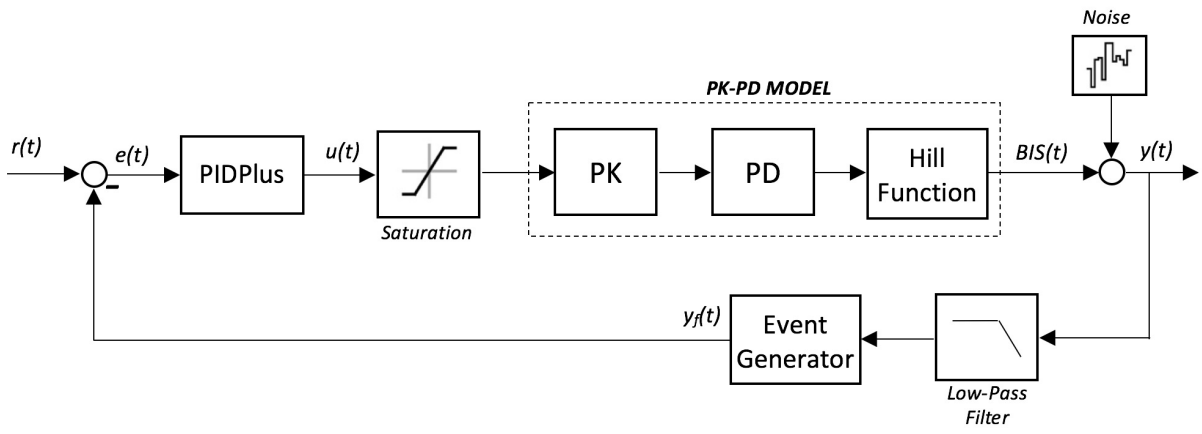

Figure 3: Event-based control structure for the propofol automatic administration

but a BIS level between 40 and 60 does not yield particular problems in the DoH of the patient.

The controller output $u(t)[\mathrm{mg} / \mathrm{s}]$ is the drug infusion rate calculated to achieve and maintain the desired BIS level. The saturation block on the control action represents the infusion rate bounds with a minimum value of $0[\mathrm{mg} / \mathrm{s}]$ and a maximum value of $6.67[\mathrm{mg} / \mathrm{s}]$. This maximum value has been calculated for a standard pump (Graseby 3400, Smiths Medical, London, UK) and it represents the maximum infusion rate with a standard propofol concentration (Diprivan $20[\mathrm{mg} / \mathrm{ml}]$ ).

The BIS signal noise has been represented as a white Gaussian additive noise on the output, which has then been filtered by a high-pass filter with a cutoff frequency of $5 \mathrm{rad} / \mathrm{s}$. Its power spectral density (PSD) has been estimated from clinical data provided by Department of Anesthesiology, Critical Care and Emergency of the Spedali Civili di Brescia University Hospital, Brescia (Italy). For all the patients the PSD of the signals has been calculated and then averaged to extract the final value of

$$
P S D=39.3392 .
$$

The event-based controller is composed of two principal blocks: the new event generator and the PIDPlus controller presented in the previous section. The new event generator has remarkable noise filtering properties and this makes it very suitable for the DoH control. As it has been mentioned before, the noise on the BIS can to be considered as band-limited Gaussian noise with zero mean. It is therefore useful to employ an integral event condition to determine the true signal change. In fact, the zero-mean noise tends to be compensated in the integral calculation. Considering only the variation between the signal and the last sample, the proposed event generation condition (that substitutes (2)) is:

$$
\left|\int_{t_{\text {last }}}^{t} y(t)-y_{f}\left(t_{\text {last }}\right) d t\right|>\Delta_{i} \text { OR } t_{w}>t_{\text {max }}
$$




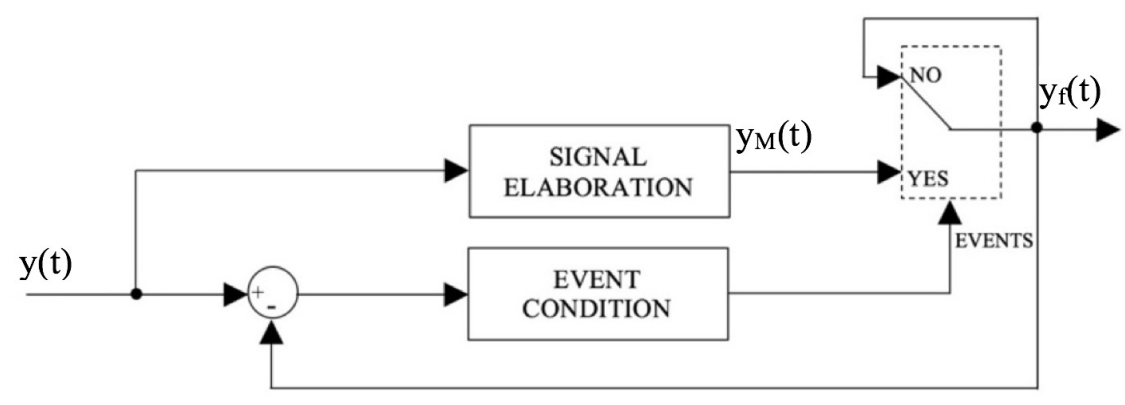

Figure 4: The Event generator structure

where $y(t)$ is the process variable, $y_{f}\left(t_{\text {last }}\right)$ is the last sample sent to the controller, $t_{\text {last }}$ is the time of the previous event, $t$ is the current time instant, $t_{w}$ is the elapsed time from the previous event, and $\Delta_{i}$ and $t_{\max }$ are the tuning parameters of the event generator. The selection of $\Delta_{i}$ allows the modification of the variation of $y(t)$ required to generate an event. The tuning of this parameter is not immediate due to the integral condition, and in the next subsection a simple procedure is proposed. The second part of the event condition is, as already explained, a safety condition that forces the triggering of the event in any case. This is useful in the maintenance phase where a set of periodical events is generated with consequent small periodical changes in infusion level to keep the required BIS level. This behavior replicates the anesthesiologist manual check and infusion regulation during the surgery. The tuning of $t_{\max }$ is also addressed in the next subsection.

In a standard SOD system, when an event occurs a sample of the process output is sent to the controller. We propose the use of another integral action to calculate the average value of the signal between the previous event and the current time instant. Thus, at each event, the average value of the BIS signal is computed as:

$$
y_{M}(t)=\frac{\int_{t_{\text {last }}}^{t} y(t) d t}{t-t_{\text {last }}} .
$$

The idea is based again on the filtering effect of the integral calculation on white noise. In the clinical practice it is also important to analyze the BIS trend rather than the exact BIS value at the event generation time instant. Averaging the process variable between two consecutive events allows the evaluation of the trend between the two considered events, replicating again the anesthesiologist behavior. The block diagram of the complete event generator is illustrated in Figure 4, where the Event Condition block implements the integral condition (6), the Signal Elaboration block calculates the average of $y(t)$ according to (7) and its calculation is kept as the event generator output $y_{f}(t)$ between two events. The resulting output $y_{f}(t)$ is therefore a piecewise constant approximation of $y(t)$ without noise. From 
a control system point of view this is a significant advantage because the filtering effect implies a great advantage in terms of the control performance and of the actuator usage.

A second-order low-pass filter is also implemented before the event generator block to filter possible high noise peaks on $y(t)$ and to prefilter the noise. The transfer function of the filter is:

$$
F(s)=\frac{1}{\left(T_{f} s+1\right)^{2}} .
$$

The filter cut-off frequency (and consequently the $T_{f}$ parameter) is tuned in order to filter only the high frequencies and to avoid modifications of the dynamics of the control loop, which might yield an excessively slow response and the risk of instability. Details on its tuning are reported in the next subsection.

\subsection{Tuning of the controller}

A correct tuning of the overall controller is necessary to obtain the desired performance and satisfy the clinical specifications. The propofol infusion has to be regulated in order to achieve a BIS level of 50 with a short settling time (about $4 \mathrm{~min}$ ) in the induction phase. In the maintenance phase the controller should reject disturbances mainly due to noxious stimuli. Several surgical disturbance profiles have been proposed in [14, 54]. All of them mainly act as additive output disturbances, thus lowering the DoH value. Some of them are quite complicated since they try to represent the arousal due to laryngoscopy, intubation, incisions, periods of no stimulations and total stimulation withdrawing. In spite of their complexity, these profiles do not actually represent exactly the reality. Hence, the disturbance model used in [51] has been considered because of its simplicity and because it allows an easy evaluation of the controller performance. It consists of a step signal of amplitude 10, acting directly on the process variable, followed by another step after 20 minutes of amplitude -10 .

In general, excessive variations of the BIS level should be avoided to reduce possible side effects. In particular, in the initial transition, it is fundamental to limit the undershoot of the BIS level as this might imply the occurrence of a dangerous hypotension [24]. A BIS range of $40-60$ is recommended from clinical practice for the maintenance phase and as a bound for the oscillation possibly induced in the initial transient. As already mentioned, both underdosing and overdosing should be avoided, as the first might yield an awakening of the patient, while the latter might yield cognitive impairment and post-operative delirium.

In the proposed control scheme six parameters have to be tuned: $K_{p}, T_{i}$ and $T_{d}$ for the PIDPlus controller, $\Delta_{i}$ and $t_{\max }$ for the event generator, and $T_{f}$ for the low-pass filter. 
In an event-based control system, there are no standard methods to tune the parameter that generates the event after a predefined time interval has elapsed without events. Indeed, the value of $t_{\max }$ should depend on the dynamics of the controlled system and on the importance of this time-based safety condition in the particular application. In the DoH control it is important to replicate the clinical practice and to guarantee an adequate safety of the patient. Based on this consideration, the value $t_{\max }=30[\mathrm{~s}]$ can be considered a reasonable time interval.

Regarding the PIDPlus parameters $K_{p}, T_{i}$ and $T_{d}$, the typical tuning rules that are generally used for this kind of controller are not suitable for the DoH control because of the process complexity. As it has been described in Section 2, the PK/PD model of propofol is a high-order system with a static nonlinearity and a linearization and/or a model reduction might result in a significant loss of information in the system dynamics. Further, also the tuning of the threshold parameter $\Delta_{i}$ is not straightforward, although its physical meaning is clear as it handles the trade-off between the filtering of the noise and the number of events.

For this reason another approach has been pursued and these parameters have been tuned by employing genetic algorithms [36], which are capable to determine a global optimum of an optimization problem in a stochastic sense. Other evolutionary algorithms could have been used for this purpose [15].

In particular, a genetic algorithm with a 100 elements of initial population and 500 generations has been used. The initial population of the parameters has been generated with a uniform distribution and a Gaussian mutation function has been used. Further, the genetic algorithm has been performed many times with different initial populations in order to be obtain a tuning which represents the global minimum. The fitness function to be minimized by the genetic algorithm has been expressed as the worst-case integrated absolute error (IAE) value by considering all the patients of the benchmark set of Table 1 (in the case where no noise is present). The integrated absolute error is defined as

$$
I A E=\int_{0}^{\infty}|r(t)-B I S(t)| d t
$$

and minimizing it generally implies a fast settling time without a large overshoot.

The obtained tuning can be regarded as an optimal robust tuning because is obtained by solving a min-max optimization problem over a set of patients representative of a large population. As a consequence, no further optimizations are required and the end user can simply adopt the parameters provided in this paper. Two different sets have been determined, one for the set-point response and one for the disturbance rejection, so that a gain scheduling control strategy can be implemented in order to improve the performance of the system [43]. 


\begin{tabular}{|c|c|c|}
\hline Parameter & Set-point & Disturbance \\
\hline$K_{p}$ & 0.0353 & 0.0215 \\
\hline$T_{i}$ & 198.86 & 320.99 \\
\hline$T_{d}$ & 25.60 & 8.38 \\
\hline$\Delta_{i}$ & 31.45 & 25.05 \\
\hline$t_{\max }$ & \multicolumn{2}{|c|}{30} \\
\hline$T_{f}$ & \multicolumn{2}{|c|}{0.1} \\
\hline
\end{tabular}

Table 2: Complete tuning of the event based system.

Finally, $T_{f}$ has to be tuned for a prefiltering of the noise, mainly to eliminate possible noise high peaks, without influencing the dynamics of the control system. Hence, the parameter has been fixed equal to 0.1 in an empirical way by taking into account the time constants of the system and after some simple simulated tests. The absence of an influence of the filter on the overall dynamics of the control system has been proven by determining (in the absence of noise) a performance decay ratio defined as

$$
d=\max _{k} \frac{I A E_{\text {filter }, k}-I A E_{k}}{I A E_{k}},
$$

where $I A E_{\text {filter }, k}$ indicates the integrated absolute error value obtained with the low-pass filter for the $k$ th patient, and $I A E_{k}$ denotes the same index calculated for the response without the prefiltering effect. This index has been calculated considering the worst case over the patients population and it results to be $0.23 \%$, so that it can be deduced that the low-pass filter does not affect the system response.

The complete tuning of the event based system is summarized in Table 2.

\section{Simulation results}

\subsection{Performance indexes}

A large number of simulations have been performed in order to test the devised event-based method. In order to evaluate the obtained performance, the following performance indexes, proposed in [20], have been calculated for the set-point following (namely, the induction phase) task:

- TT: observed time-to-target (in seconds) required for reaching the first time the target interval of 45-55 BIS values;

- BIS-NADIR: the lowest observed BIS value;

- ST10: settling time, defined as the time interval for the BIS to reach and steady within the BIS range between 45 and 55 (that is, the target value of $50 \pm 5$ ); 

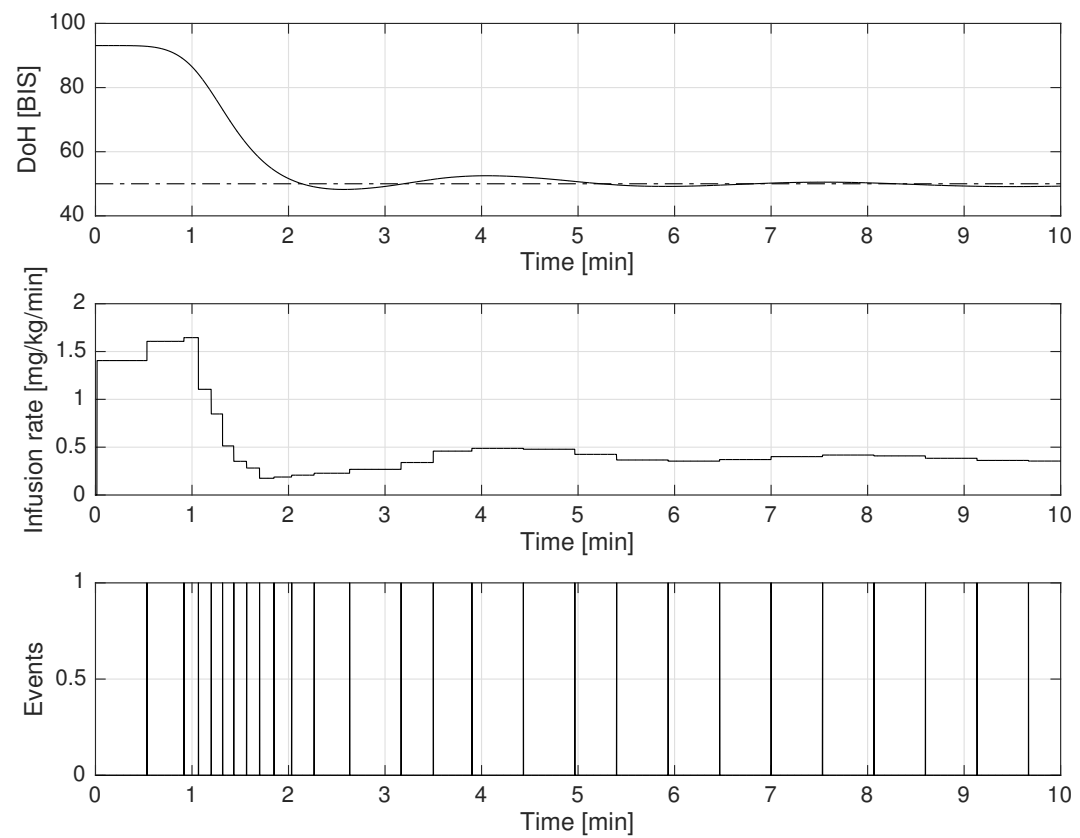

Figure 5: Set-point response of the average patient 13. Top plot: BIS level. Middle plot: event-based control action. Bottom plot: events generated.

- ST20: the same of ST10 but it considers a BIS range of 40 and 60;

- US: undershoot, defined as the difference between the lower threshold of 45 and the minimum value of BIS below this threshold.

Regarding the load disturbance rejection task, that is the maintenance phase, only the TT and the BIS-NADIR indexes are meaningful, by taking into account that the disturbance signal consists of a step signal of amplitude 10, acting directly on the process variable, followed by another step after 20 minutes of amplitude -10. However, it is sensible to calculate the indexes separately for the positive and for the negative step.

\subsection{Average patient}

As a first simulation result, the induction phase of the thirteen (average) patient is plotted in Figure 5 in order to illustrate the rationale of the method. The BIS signal is shown in the first plot, the event-based control action in the second and the events generated by the noise filtering event generator in the third. The resulting performance indexes are shown in Table 3. It appears that the achieved performance is satisfactory from a clinical point of view as the required $\mathrm{DoH}$ is attained in a short time without undershoot. 


\begin{tabular}{|c|c|}
\hline TT [min] & 1.81 \\
\hline BIS-NADIR & 48.23 \\
\hline ST10 [min] & 1.81 \\
\hline ST20 [min] & 1.64 \\
\hline US & 0 \\
\hline
\end{tabular}

Table 3: Performance indexes of the step-response for the average patient 13 .

In particular, it can be noted that TT is equal to ST10 and this proves that there is no undershoot over the 45-55 range. The settling time should be interpreted by also taking into account that the proposed control systems regulates the infusion in the initial transition without using a bolus. While this implies a slight increment of the settling time, it also avoids a dangerous undershoot in BIS level [44]. Further, the avoidance of the initial rapid bolus reduces the risk of hemodynamic instability with vascular dilation and stroke volume reduction with harmful hypotension [57]. By analyzing the control variable, it can be observed that the infusion rate is piecewise constant because of the event-based strategy. Indeed, the controller keeps $u(t)$ at a constant value until a BIS significant change occurs with clinical benefits due to the more regular infusion. This kind of infusion has advantages in the patient hemodynamics compared to the continuous control action variation obtained with other control strategies. From a technical point of view, a piecewise constant control variable guarantees also less pump stress. Another very relevant advantage of the event-based strategy is the total absence of the noise in the control variable thanks to the filtering properties of the devised event generator. This is indeed beneficial for the pump because there are no useless variations. In order to highlight the filtering properties of the event generator, the (noisy) process variable $y(t)$ is plotted together with the output of the event generator $y_{f}(t)$ (that is, the actual feedback signal) in Figure 6. It clearly appears that $y_{f}(t)$ is not affected by a residual noise as it usually happens with standard low-pass filtering techniques. By analyzing the events that are generated during the experiment of Figure 5, it is possible to observe that more events occur during the transient because of the rapid variation of the BIS level. Then, when the BIS is close to the reference value, the safety synchronous condition generates events every 30 $[\mathrm{s}]$, that is, before the triggering of the integral condition. This forces small corrections of the control action to achieve the BIS reference value.

The excellent filtering properties of the scheme are confirmed in Figure 7 where, again for patient 13, two simulations, one with noise and the other one without noise are compared. Results are virtually the same, demonstrating the effectiveness of the event generator. 


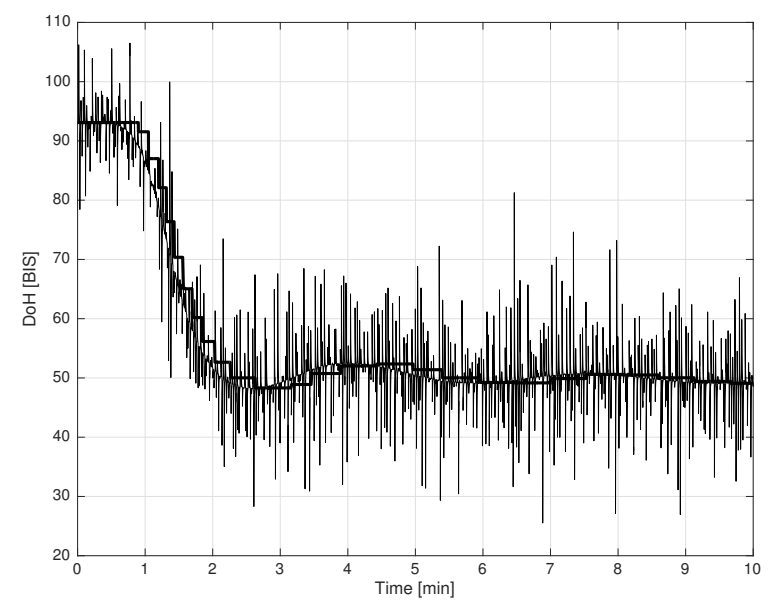

Figure 6: Noisy process variable $y(t)$ and the corresponding event generator output $y_{f}(t)$ (thick solid line).
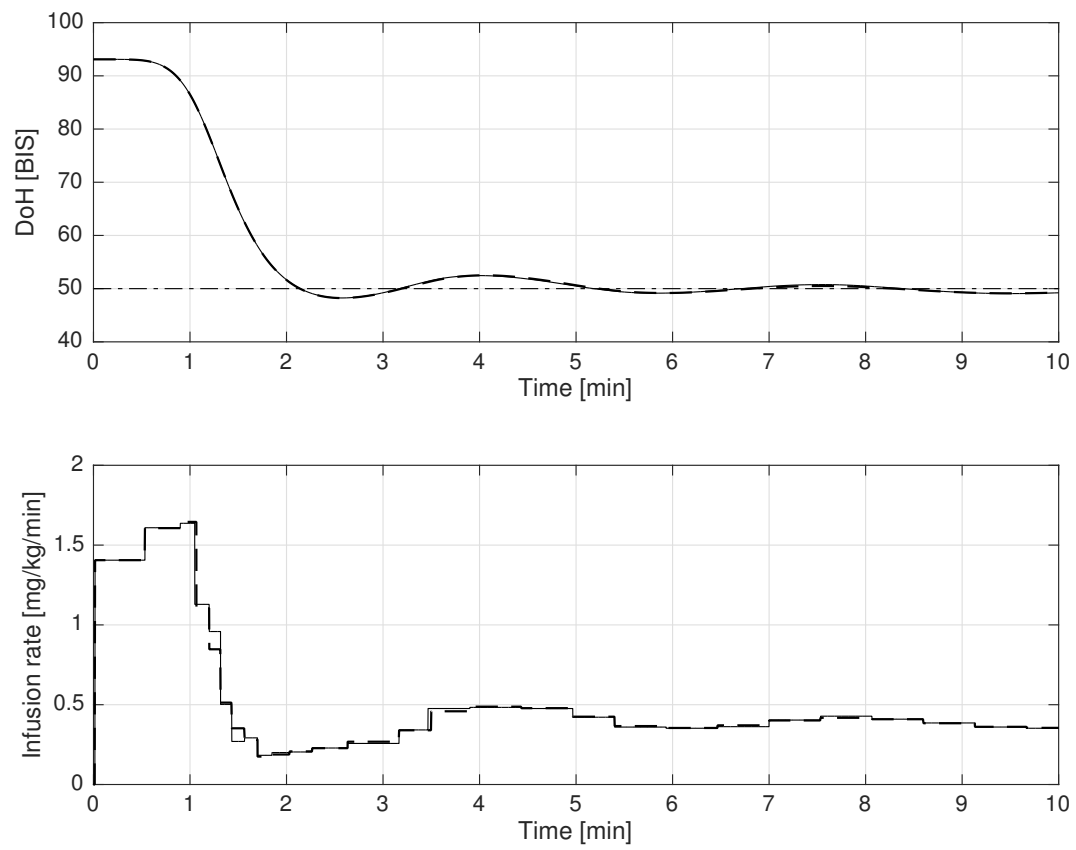

Figure 7: BIS signal and control action in the induction phase with (solid line) and without (dashed line) measurement noise.

Two additional performance indexes have been considered, the Integrated Absolute Error defined in (9), which evaluates the tracking perfor- 


\begin{tabular}{|c|c|c|c|c|}
\hline \multirow{2}{*}{ Index } & \multicolumn{2}{|c|}{ Set-point } & \multicolumn{2}{c|}{ Disturbance } \\
\cline { 2 - 5 } & With noise & Without noise & With noise & Without noise \\
\hline IAE & 3947.2 & 3932.9 & 2062.7 & 2049.4 \\
\hline TV & 4.03 & 4.00 & 2.63 & 2.46 \\
\hline
\end{tabular}

Table 4: Comparison of IAE and TV of the set-point and disturbance rejection response of average patient 13 with and without noise.

\begin{tabular}{|c|c|c|c|}
\hline TTp [min] & 0.63 & TTn [min] & 1.14 \\
\hline BIS-NADIRp & 47.39 & BIS-NADIRn & 52.44 \\
\hline
\end{tabular}

Table 5: Disturbance rejection indexes for the average patient 13.

mance of the controller, and the Total Variation $(T V)$ defined as

$$
T V=\sum_{k=0}^{\infty}\left|u_{k}-u_{k-1}\right|,
$$

where $u_{k}$ is the current control action value and $u_{k-1}$ is the previous one. Index (11) evaluates the control action variation and therefore the overall control effort. These indexes have been calculated in a simulation of 10 [min] with a sampling period of $1[\mathrm{~s}]$ and the resulting values are shown in Table 4 .

Another test performed on the system is related to the maintenance phase, that is, to the disturbance rejection task, in order to evaluate the compensation of possible noxious stimuli. Results for patient 13 are shown in Figure 8. As for the induction phase, also in this case the control action is modified by a sequence of events. In particular, the controller increases the drug infusion with the first (positive) step to decrease the DoH of the patient and viceversa with the second (negative) step. The same indexes proposed for the set-point response are evaluated for each step in Table 5. Time intervals are calculated from the time instant of the application of the corresponding step disturbance. The indexes for the positive step are denoted with the letter ' $p$ ' and for the negative step with letter ' $n$ ' respectively. As already mentioned, the other indexes are not shown as they are meaningless for this task. It appears that the settling times are satisfactory according to the clinical practice as the controller ensures a disturbance rejection with limited effects on DoH of the patient. The TTn value is higher than the TTp value because of the saturation of the pump. In fact, the controller has to decrease the infusion level to compensate the decrement of the BIS level, but the lower infusion limit is zero. The pump is therefore switched off until the BIS level naturally increases and this produces a higher settling time. As for the induction phase, the efficacy of the filter is then verified by comparing the noisy and noise-free cases. Results shown in Figure 9 show that 

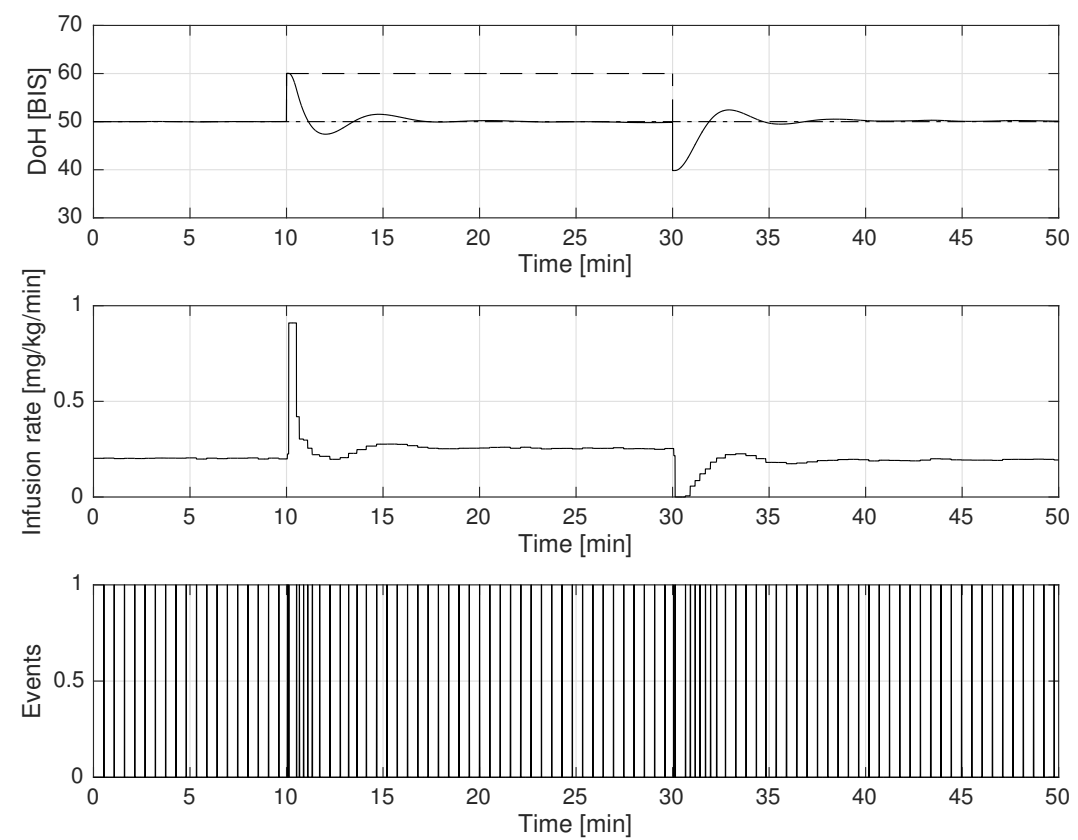

Figure 8: Disturbance rejection evaluated for the average patient 13. Top plot: BIS level. Middle plot: event-based control action. Bottom plot: events generated.

the responses in the two cases again are almost overlapped. The IAE and TV indexes have been calculated also in this case in a simulation of 50 [min] with a sampling period of $1[\mathrm{~s}]$ and their values are reported in Table 4, where the effectiveness of the event generator appears also in the disturbance rejection case.

\subsection{Robustness evaluation}

The robustness of the controller has to be validated by considering the interpatient variability and for this purpose the same tests performed on the average patient 13 have been initially performed on the entire population of Table 1. The set-point responses (induction phase) are shown in Figure 10. The trends of the BIS level and the control actions are quite similar for each patient. All the performance indexes have been calculated also in this case and they are reported in Table 6. It is worth stressing that TT is always equal to ST10 and US is always zero. In Figure 11 the disturbance rejection case is shown for each patient and the corresponding indexes are reported in Table 7. Settling times are again satisfactory with respect to the clinical 

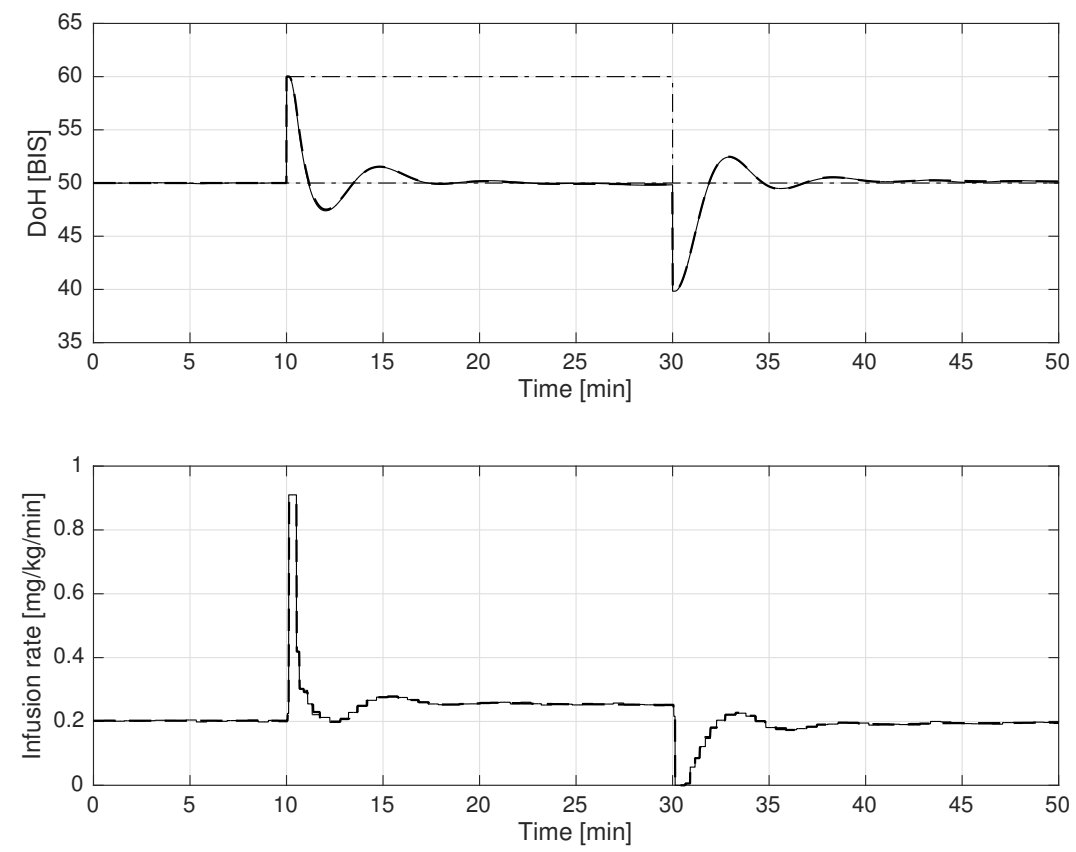

Figure 9: BIS signal and control action in the maintenance phase with (solid line) and without (dashed line) measurement noise.

practice as the oscillations of the BIS level are always bounded in an safety interval.

The inter-patient robustness has been further tested by implementing a Monte Carlo method where 500 PK-PD models of patients have been generated by considering a uniform distribution of the age between 18 and 70, of the height between $150[\mathrm{~cm}]$ and $190[\mathrm{~cm}]$, and of the weight between $50[\mathrm{~kg}]$ and $100[\mathrm{~kg}]$. Then, the distribution of the values for the Hill function parameters have been taken from [56]. Results related to the induction phase are shown in Figure 12, while those related to the maintenance phase are shown in Figure 13. The corresponding indexes are shown in Tables 8 and 9. The controller is robust to the inter-patient variability as all the clinical specifications are always fulfilled. From another point of view, it is confirmed that the patients of Table 1 of the paper are really representative of a wide population, as the controller tuned by only taking them into account can be satisfactorily applied to a general population.

Finally, we consider intra-patient variability which is the statistical distribution of the model parameters. Another Monte Carlo method has been implemented. In particular, for each patient in Table 1, a set of 1000 models have been generated based on the statistical properties of the model 

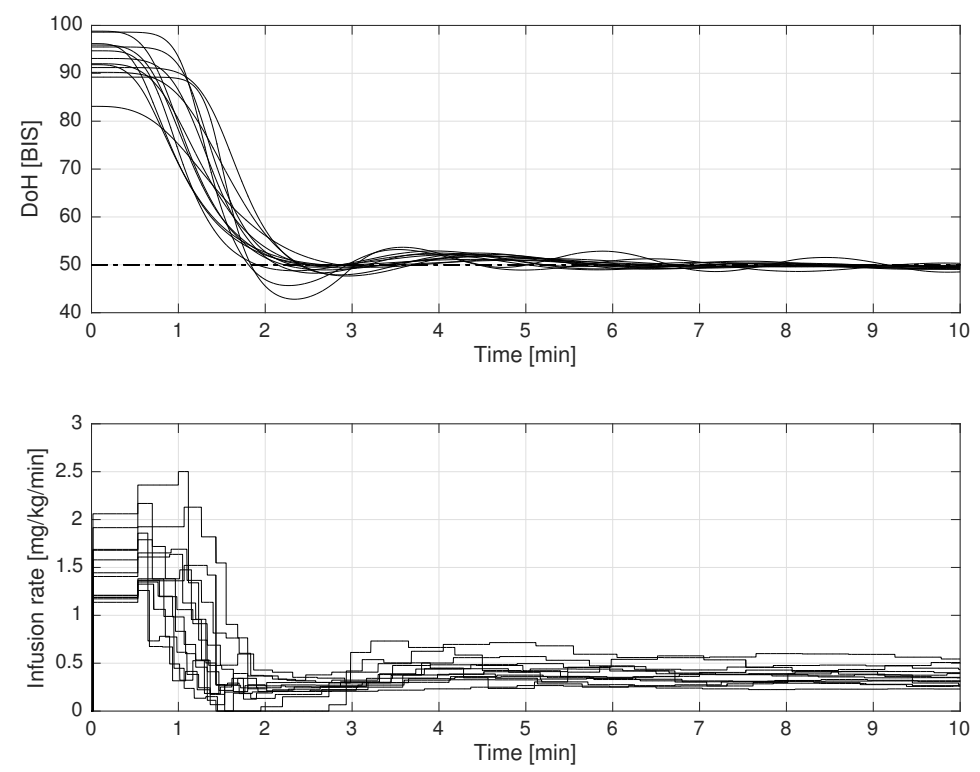

Figure 10: BIS level and control action (induction phase) of each patient of the data set.

\begin{tabular}{|c|c|c|c|c|c|}
\hline Patient & TT $[\mathrm{min}]$ & BIS-NADIR & ST20 $[\mathrm{min}]$ & ST10 $[\mathrm{min}]$ & US \\
\hline 1 & 1.73 & 48.94 & 1.49 & 1.73 & 0 \\
\hline 2 & 1.64 & 45.67 & 1.53 & 1.64 & 0 \\
\hline 3 & 2.09 & 47.91 & 1.92 & 2.10 & 0 \\
\hline 4 & 1.76 & 49.58 & 1.49 & 1.76 & 0 \\
\hline 5 & 1.51 & 48.76 & 1.32 & 1.51 & 0 \\
\hline 6 & 2.07 & 47.67 & 1.85 & 2.07 & 0 \\
\hline 7 & 1.90 & 49.39 & 1.64 & 1.90 & 0 \\
\hline 8 & 1.87 & 49.38 & 1.68 & 1.87 & 0 \\
\hline 9 & 1.72 & 42.81 & 1.64 & 2.70 & 2.19 \\
\hline 10 & 2.09 & 49.50 & 1.78 & 2.09 & 0 \\
\hline 11 & 1.73 & 49.65 & 1.40 & 1.73 & 0 \\
\hline 12 & 1.68 & 49.68 & 1.38 & 1.68 & 0 \\
\hline 13 & 1.82 & 48.25 & 1.64 & 1.82 & 0 \\
\hline Mean & 1.82 & 48.24 & 1.59 & 1.89 & 0.17 \\
\hline Std. Dev. & 0.18 & 1.99 & 0.18 & 0.30 & 0.61 \\
\hline Min & 1.51 & 42.81 & 1.32 & 1.51 & 0 \\
\hline Max & 2.09 & 49.68 & 1.92 & 2.70 & 2.19 \\
\hline
\end{tabular}

Table 6: Performance indexes for the set-point response of each patient. 

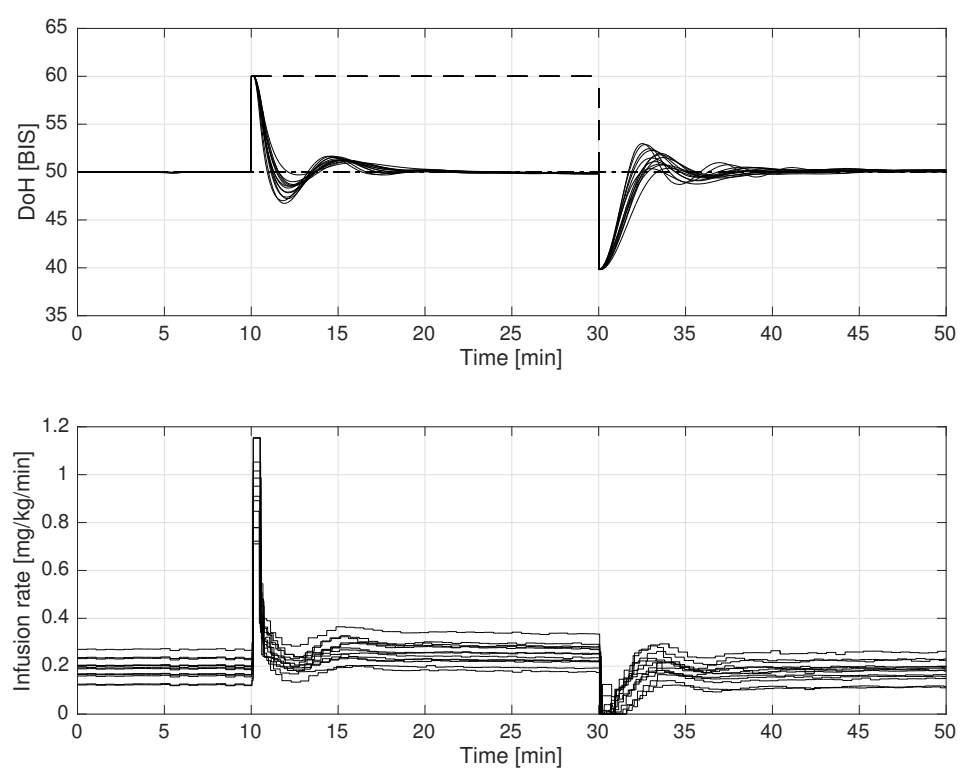

Figure 11: BIS level and control action (maintenance phase) of each patient of the data set.

\begin{tabular}{|c|c|c|c|c|}
\hline Patient & TTp [min] & BIS-NADIRp & TTn [min] & BIS-NADIRn \\
\hline 1 & 0.74 & 48.59 & 1.45 & 51.80 \\
\hline 2 & 0.58 & 46.74 & 1.08 & 52.87 \\
\hline 3 & 0.66 & 47.90 & 1.20 & 51.47 \\
\hline 4 & 0.68 & 48.33 & 1.18 & 50.36 \\
\hline 5 & 0.65 & 47.85 & 1.54 & 51.95 \\
\hline 6 & 0.68 & 47.23 & 1.15 & 52.26 \\
\hline 7 & 0.73 & 48.51 & 1.37 & 50.82 \\
\hline 8 & 0.65 & 48.53 & 1.36 & 51.09 \\
\hline 9 & 0.53 & 47.01 & 1.10 & 52.98 \\
\hline 10 & 0.79 & 48.70 & 1.43 & 50.74 \\
\hline 11 & 0.82 & 49.70 & 1.76 & 50.40 \\
\hline 12 & 0.74 & 48.99 & 1.57 & 51.51 \\
\hline 13 & 0.64 & 47.34 & 1.14 & 52.44 \\
\hline Mean & 0.68 & 48.12 & 1.33 & 51.59 \\
\hline Std. Dev. & 0.08 & 0.86 & 0.21 & 0.89 \\
\hline Min & 0.53 & 46.74 & 1.07 & 50.36 \\
\hline Max & 0.82 & 49.70 & 1.76 & 52.98 \\
\hline
\end{tabular}

Table 7: Performance indexes for the disturbance rejection of each patient. 

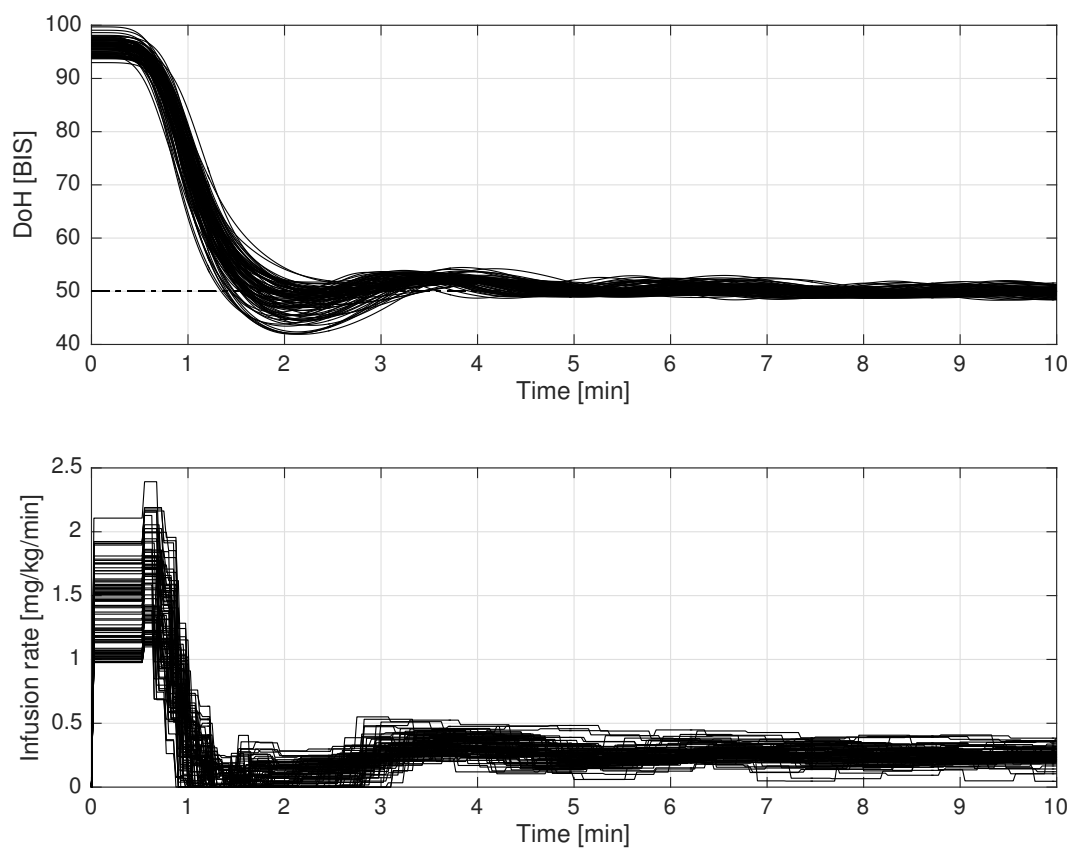

Figure 12: Set-point step responses by using Monte Carlo method to verify the controller robustness with respect to the inter-patient variability.

\begin{tabular}{|c|c|c|c|c|c|}
\hline Index & TT $[\mathrm{min}]$ & BIS-NADIR & ST10 $[\mathrm{min}]$ & ST20 $[\mathrm{min}]$ & US \\
\hline Mean & 1.48 & 47.42 & 1.69 & 1.32 & 0.30 \\
\hline Std. Dev. & 0.15 & 2.33 & 0.41 & 0.15 & 0.91 \\
\hline Min & 1.13 & 38.90 & 1.25 & 1.07 & 0 \\
\hline Max & 1.94 & 49.88 & 2.85 & 2.34 & 6.1 \\
\hline
\end{tabular}

Table 8: Performance indexes for the set point responses with the Monte Carlo method for inter-patient variability.

\begin{tabular}{|c|c|c|c|c|}
\hline Index & TTp [min] & BIS-NADIRp & TTn [min] & BIS-NADIRn \\
\hline Mean & 0.62 & 47.29 & 1.26 & 52.39 \\
\hline Std. Dev. & 0.02 & 1.01 & 0.13 & 1.03 \\
\hline Min & 0.50 & 44.48 & 1.01 & 50.03 \\
\hline Max & 0.77 & 49.81 & 1.57 & 54.41 \\
\hline
\end{tabular}

Table 9: Performance indexes for the load disturbance step responses with the Monte Carlo method for inter-patient variability. 

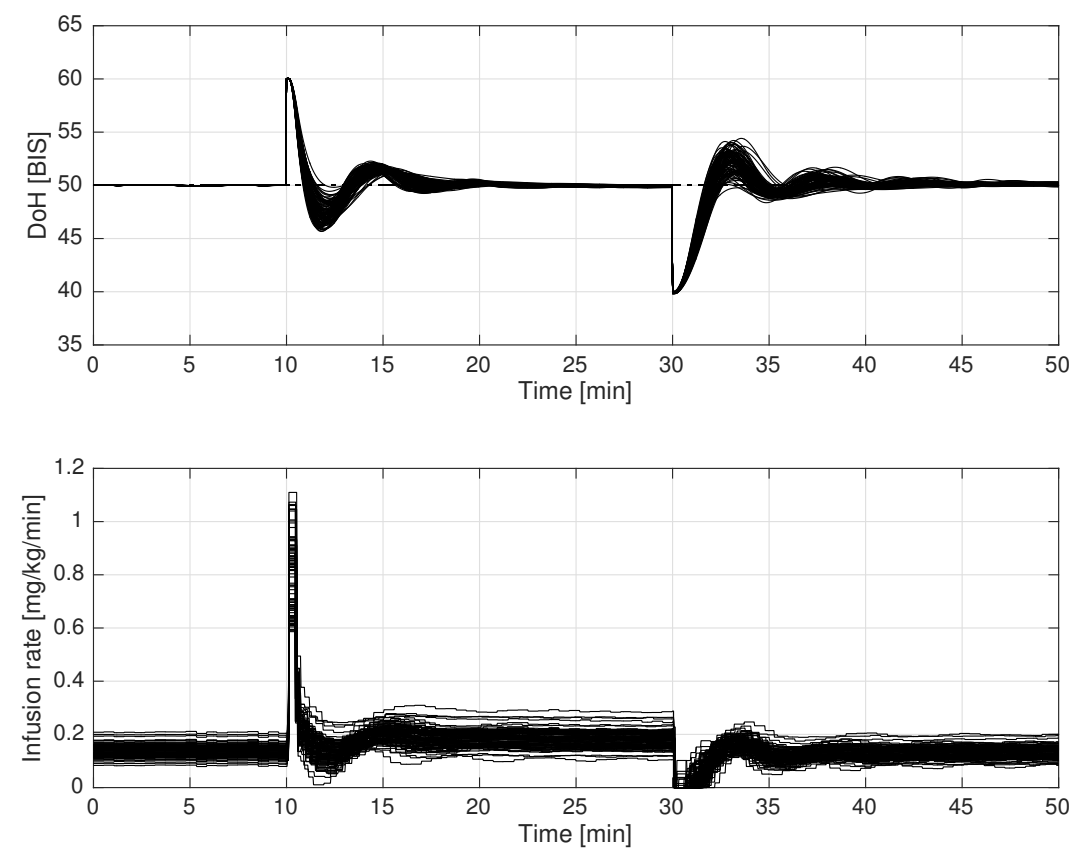

Figure 13: Load disturbance step responses by using Monte Carlo method to verify the controller robustness with respect to the inter-patient variability. 

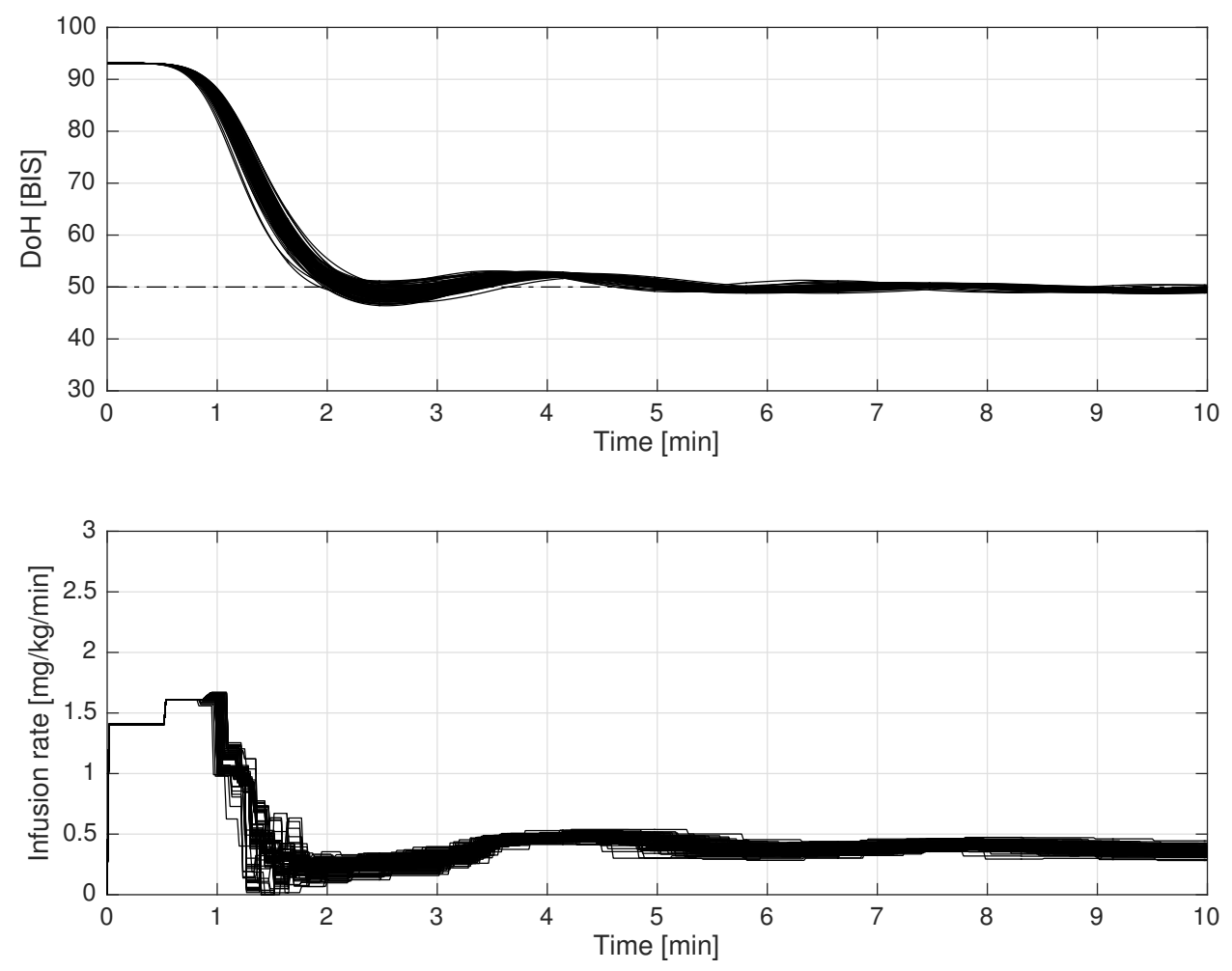

Figure 14: Set-point step responses by using Monte Carlo method to verify the controller robustness with respect to the intra-patient variability (average patient 13).

reported in [49]. For the sake of readability, results related only to the average patient 13 are shown in Figure 14 and the corresponding performance indexes are reported in Table 10. Despite the intra-patient variability, the set-point response with the event-based controller is always satisfactory and the clinical specification are always fulfilled. Obviously, there is some variability in the calculated indexes but, as it shown in Table 14, it is limited and does not significantly influence the induction phase. The results of the Monte Carlo method for all the patients in the database are shown in Figure 15. It can be deduced that the robustness of the controller is confirmed for all the patients.

The same considerations can be done for the disturbance rejection task. The corresponding results, again related to 1000 models generated with the Monte Carlo method for the average patient 13, are shown in Figure 16 and the performance indexes are reported in Table 11, while the results for all the patients are shown in Figure 17. 

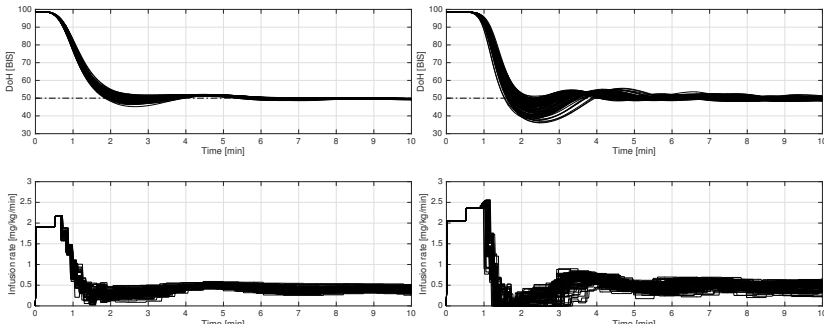

(a) Patient 1

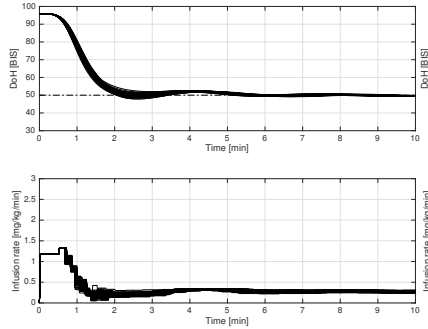

(d) Patient 4

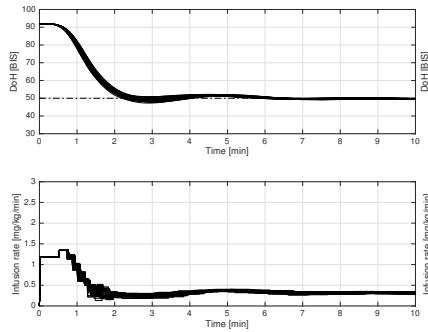

(g) Patient 7
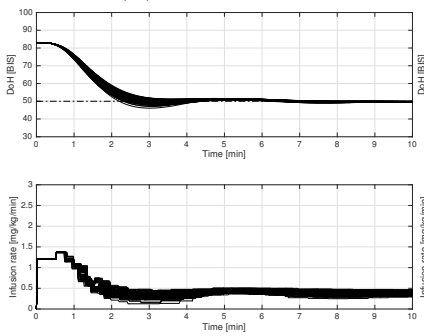

(j) Patient 10

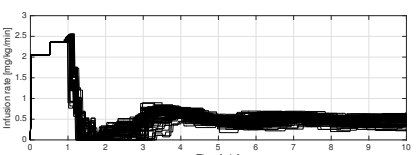

(b) Patient 2
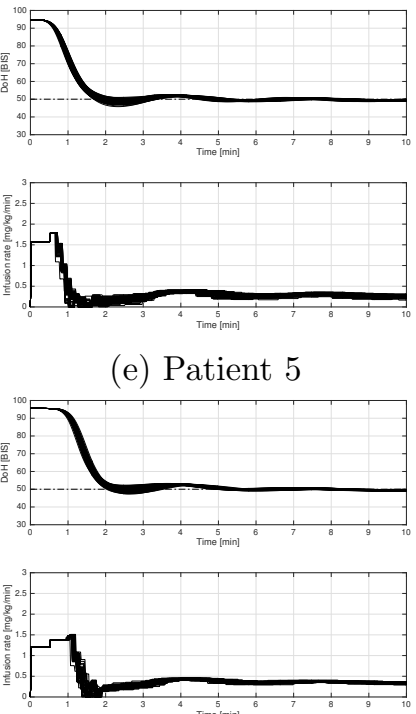

(h) Patient 8
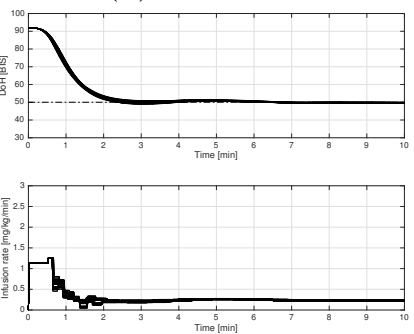

(k) Patient 11 (e) Patient 5
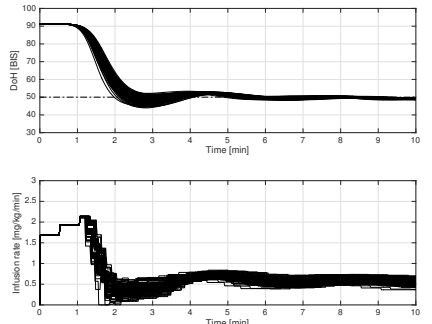

(c) Patient 3
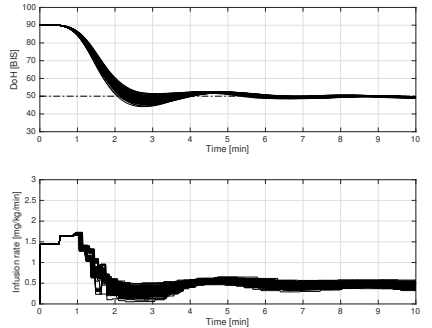

(f) Patient 6
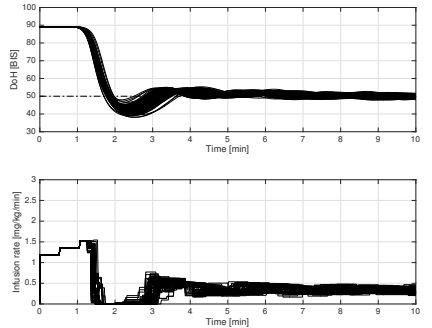

(i) Patient 9
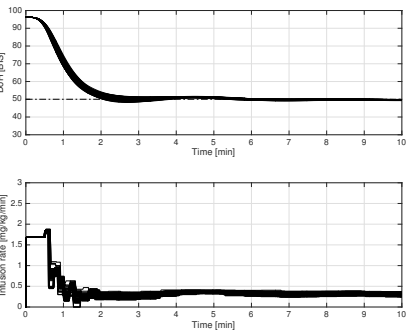

(l) Patient 12

Figure 15: Monte Carlo method results for the set point step response for all the patients. 


\begin{tabular}{|c|c|c|c|c|c|}
\hline Index & TT $[\mathrm{min}]$ & BIS-NADIR & ST10 [min] & ST20 [min] & US \\
\hline Mean & 1.84 & 48.47 & 1.84 & 1.64 & 0 \\
\hline Std. Dev. & 0.07 & 0.82 & 0.07 & 0.06 & 0 \\
\hline Min & 1.63 & 46.39 & 1.63 & 1.47 & 0 \\
\hline Max & 2.03 & 49.54 & 2.03 & 1.80 & 0 \\
\hline
\end{tabular}

Table 10: Performance indexes for the set point responses with the Monte Carlo method for intra-patient variability (average patient 13).
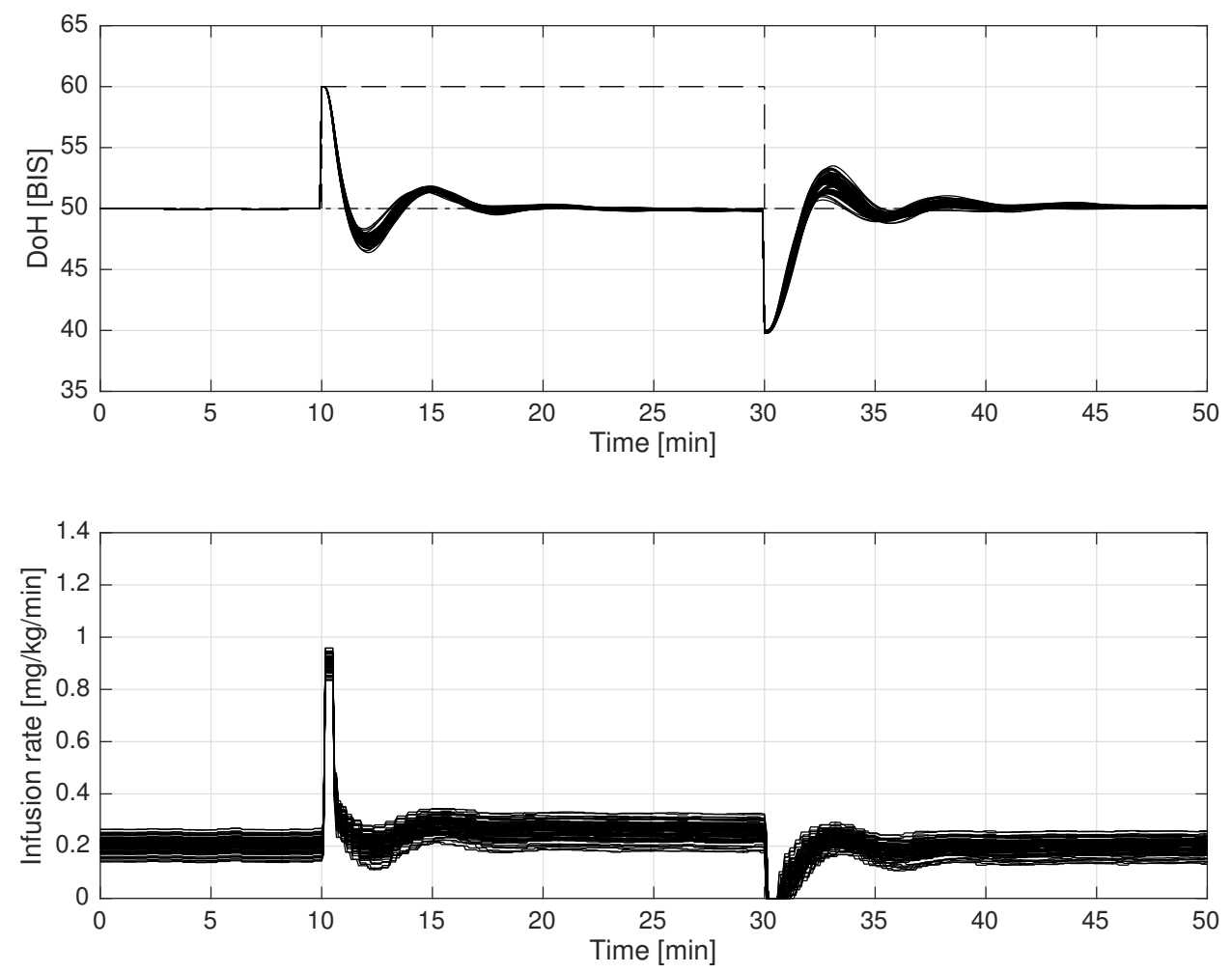

Figure 16: Load disturbance step responses by using Monte Carlo method to verify the controller robustness with respect to the intra-patient variability (average patient 13).

\subsection{Comparison with a standard PID controller}

In this section the event-based control system proposed in the paper is compared with a standard (time-driven) PID control system, which has been widely investigated and employed also in clinical trials. For this purpose, the data set of Table 1 is considered.

The standard control scheme is shown in Figure 18 where a low-pass filer is implemented on the BIS signal to eliminate the noise peaks. Then, an 


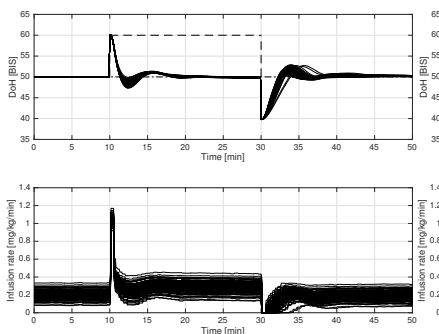

(a) Patient 1

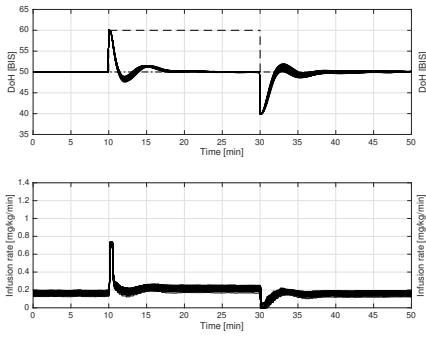

(d) Patient 4

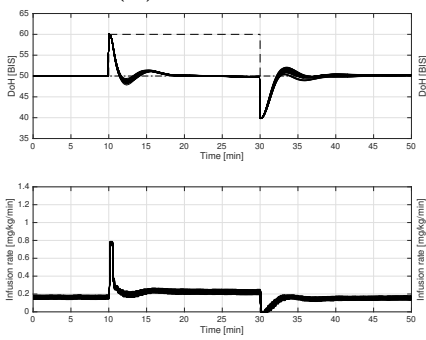

(g) Patient 7

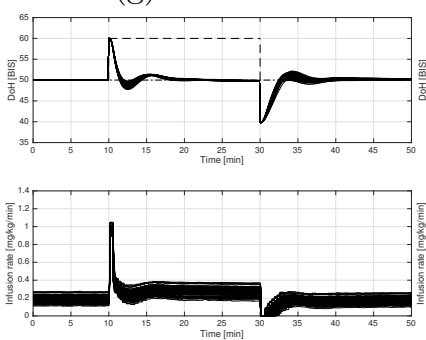

(j) Patient 10
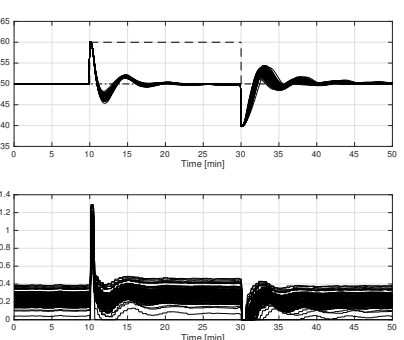

(b) Patient 2
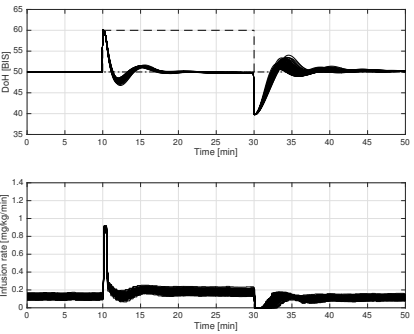

(e) Patient 5
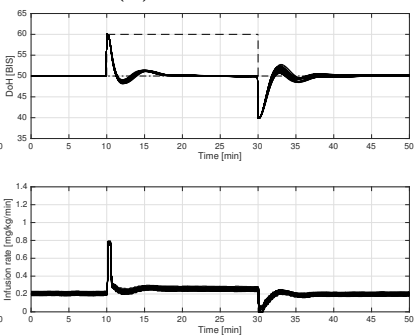

(h) Patient 8
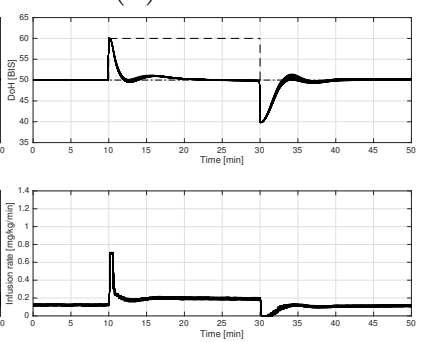

(k) Patient 11
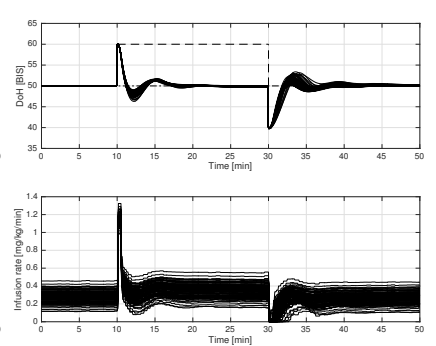

(c) Patient 3
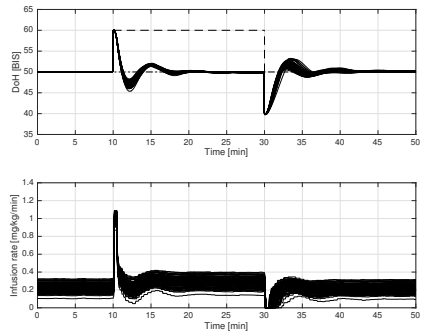

(f) Patient 6
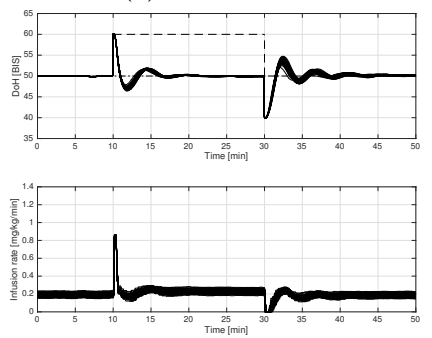

(i) Patient 9
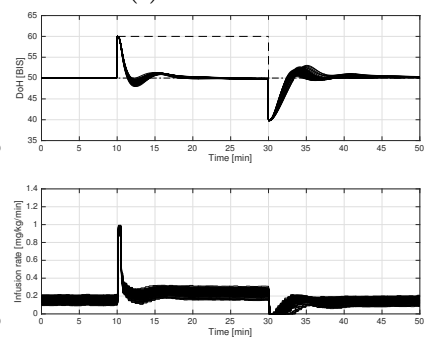

(l) Patient 12

Figure 17: Monte Carlo method results for the load disturbance step response for all the patients. 


\begin{tabular}{|c|c|c|c|c|}
\hline Index & TTp [min] & BIS-NADIRp & TTn [min] & BIS-NADIRn \\
\hline Mean & 0.65 & 47.44 & 1.14 & 52.21 \\
\hline Std. Dev. & 0.02 & 0.37 & 0.06 & 0.55 \\
\hline Min & 0.61 & 46.38 & 0.99 & 51.24 \\
\hline Max & 0.69 & 48.34 & 1.30 & 53.50 \\
\hline
\end{tabular}

Table 11: Performance indexes for the load disturbance step responses with the Monte Carlo method for intra-patient variability (average patient 13).

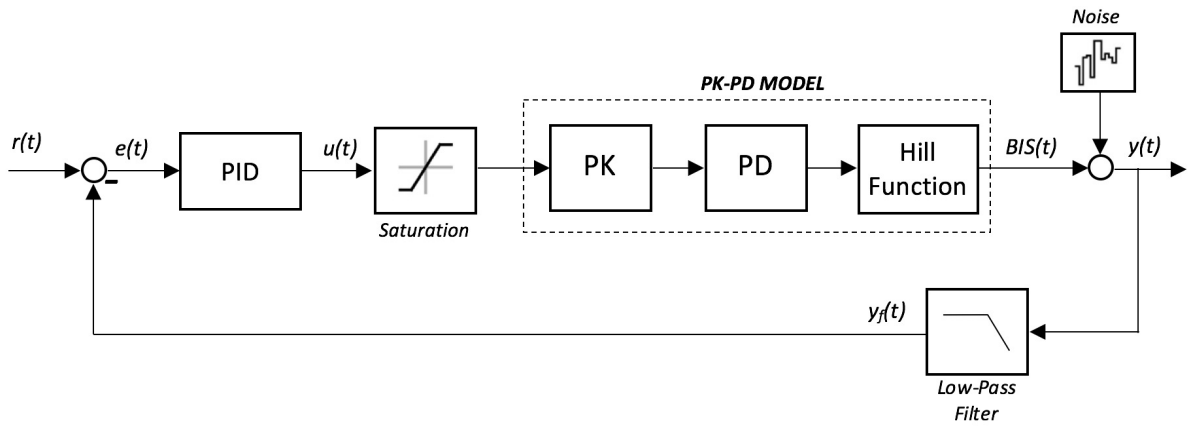

Figure 18: Standard PID control structure.

output-filtered PID controller is used. Its transfer function is expressed as

$$
C(s)=K_{p}\left(1+\frac{1}{s T_{i}}+s T_{d}\right) \frac{1}{\left(s T_{f 2}+1\right)^{2}},
$$

where $K_{p}$ is the proportional gain, $T_{i}$ is the integral time constant, $T_{d}$ is the derivative time constant and $T_{f 2}$ is the time constant of the low-pass filter used to reduce the noise on the control variable. This control system and its tuning has been already investigated in [43]. In order to perform a fair comparison, the tuning of the PID controller has been determined again with genetic algorithms by minimizing the IAE, and the set-point response and the load disturbance rejection tasks have been considered separately. The value of $T_{f 2}$ has been determined by achieving a performance decay ratio (10) of $20 \%$, which is a decrement of performance that can be accepted in order to have a reasonable filtering of the noise. The complete tuning of the system is reported in [43] and $T_{f}$ is fixed to 0.1 as for the event-based control system. The controller has been implemented in a discrete-time version and the sampling period has been selected to $1[\mathrm{~s}]$.

The set-point responses and the disturbance rejection response of all the patients of Table 1 for the standard PID control system are shown in Figure 19. This control system satisfies the clinical specifications, with fast response and reduced overshoot of the BIS level in each case. However, the 


\begin{tabular}{|c|c|c|c|c|c|c|c|c|c|c|}
\hline \multirow{2}{*}{ Index } & \multicolumn{2}{|c|}{ TT $[\mathrm{min}]$} & \multicolumn{2}{c|}{ BIS-NADIR } & \multicolumn{2}{c|}{ ST20 [min] } & \multicolumn{2}{c|}{ ST10 [min] } & \multicolumn{3}{c|}{ US } \\
\cline { 2 - 11 } & PI & PID & PI & PID & PI & PID & PI & PID & PI & PID \\
\hline Mean & 1.89 & $\mathbf{1 . 2 7}$ & 42.02 & $\mathbf{4 7 . 5 3}$ & 2.39 & $\mathbf{1 . 2 5}$ & 5.23 & $\mathbf{1 . 5 2}$ & 3.33 & $\mathbf{1 . 1 5}$ \\
\hline Std. Dev. & 0.27 & 0.16 & 3.98 & 4.52 & 1.35 & 0.45 & 1.49 & 0.51 & 3.50 & 2.87 \\
\hline Min & 1.52 & 1.01 & 33.94 & 35.99 & 1.38 & 0.86 & 1.63 & 1.01 & 0 & 0 \\
\hline Max & 2.31 & 1.49 & 48.77 & 49.83 & 5.38 & 2.39 & 6.34 & 2.70 & 11.06 & 9.01 \\
\hline
\end{tabular}

(a) Set-point response

\begin{tabular}{|c|c|c|c|c|c|c|c|c|}
\hline \multirow{2}{*}{ Index } & \multicolumn{2}{|c|}{ TTp $[\mathrm{min}]$} & \multicolumn{2}{c|}{ BIS-NADIRp } & \multicolumn{2}{c|}{ TTn $[\mathrm{min}]$} & \multicolumn{2}{c|}{ BIS-NADIRn } \\
\cline { 2 - 9 } & PI & PID & PI & PID & PI & PID & PI & PID \\
\hline Mean & 0.52 & $\mathbf{0 . 4 6}$ & 46.55 & $\mathbf{4 8 . 3 9}$ & 1.16 & $\mathbf{1 . 1 4}$ & 52.01 & $\mathbf{5 1 . 1 1}$ \\
\hline Std. Dev. & 0.06 & 0.06 & 0.75 & 0.45 & 0.22 & 0.22 & 0.59 & 0.28 \\
\hline Min & 0.40 & 0.35 & 45.39 & 47.66 & 0.88 & 0.88 & 51.21 & 50.72 \\
\hline Max & 0.62 & 0.57 & 48.03 & 49.27 & 1.57 & 1.58 & 53.25 & 51.70 \\
\hline
\end{tabular}

(b) Disturbance rejection

Table 12: Performance indexes for the set-point response and disturbance rejection of each patient with standard PID and PI controller

main problem of this approach is the residual noise on the control action as it is shown in Figure 19. In fact, the derivative action of the controller amplifies the measurement noise and the low-pass filter on the control action can not entirely compensate for this effect. This may be a problem for the infusion pump. The problem is more evident in the set-point response due to the higher value of $T_{d}$ and consequentially of the derivative action.

A simple solution is to remove the derivative action from the controller by using a PI controller with a second-order low-pass filter on the control action. In this way the measurement noise is not amplified from the controller and the filter can better compensate for the noise. A new tuning of the system is necessary and this can be done by using the same criteria employed for the PID controller. The resulting controller parameters are also reported in [43]. As it is shown in Figure 20, this solution compensates for the measurement noise on the control action, however the performance obtained is not as satisfactory as in the PID case, since a longer settling time and a higher undershoot result. In fact, even if a correct $\mathrm{DoH}$ is guaranteed in a reasonable time, oscillations occur because of the absence of the derivative action. In the disturbance rejection task a small residual noise is still present because of the aggressive tuning of the controller. The comparison of the performance indexes for the standard PID and PI controller is shown in Table 12.

It can be observed that TT, ST20, and (most of all) ST10 are higher in the PI case. The oscillatory trend of the BIS level is detected by the 
US index that, in the PI case, is seven times more than the PID case. The minimum BIS value with the PI controller is 34.62 that can be too low from a clinical point of view. The same result applies for disturbance rejection indexes.

On the contrary, the event-based control system compensates for the noise thanks to the new event generator and the derivative action can be fully exploited. The event-based paradigm allows both a small settling time and a bounded undershoot of the BIS level, close to those obtained with the standard PID controller, see Figure 21, but it completely rejects the noise. The main difference between the responses obtained with the two systems is at the beginning of the transient response. In this phase the standard PID has a faster reaction in comparison with the event-based system (in the latter case TT increases of $15 \%$ and ST10 and ST20 of 6\%). This effect does not imply significant differences from a clinical point of view. In order to provide a deeper analysis, the indexes presented in [54] are evaluated to quantitatively compare the performance of the two control systems. In particular, this analysis is performed for a simulation of 10 [min] for setpoint response and of 50 [min] for the disturbance rejection response, with a sampling period of $1[\mathrm{~s}]$. The considered performance indexes are:

- PE: performance error, calculated for each patient $i$ and for each sample $j$ according to the formula

$$
P E_{i j}=\frac{B I S_{j}(t)-\overline{B I S}}{\overline{B I S}} \cdot 100 \quad j=1, \ldots, N_{i},
$$

where $\overline{B I S}$ is the reference BIS value and $B I S$ is the measured one;

- MDPE: median performance error, which is a measure of bias and describes whether the measured values are systematically distributed either above or below the BIS reference. It is calculated as:

$$
M D P E_{i}=\operatorname{Median}\left\{P E_{i j}, \quad j=1, \ldots, N_{i}\right\},
$$

where $j$ is the measured sample and $N_{i}$ is the number of PE values obtained for the $i$ th patient;

- MDAPE: median absolute performance error, which reflects the inaccuracy of the control method for the $i$ th patient; it is defined as

$$
M D A P E_{i}=\operatorname{Median}\left\{\left|P E_{i j}\right|, \quad j=1, \ldots, N_{i}\right\} ;
$$

- WOBBLE, which is an index of time-related changes in the performance and measures the intra-patient variability in performance errors as:

$$
W O B B L E_{i}=\operatorname{Median}\left\{\left|P E_{i j}-M D A P E_{i}\right|, \quad j=1, \ldots, N_{i}\right\} .
$$




\begin{tabular}{|c|c|c|c|c|}
\hline \multirow{2}{*}{ Index } & \multicolumn{2}{|c|}{ Set-point } & \multicolumn{2}{c|}{ Disturbance } \\
\cline { 2 - 5 } & PID standard & PID events & PID standard & PIDevents \\
\hline MDPE $[\%]$ & $\mathbf{0 . 4 6} \pm \mathbf{1 . 1 6}$ & $0.61 \pm 0.51$ & $\mathbf{0 . 1 6} \pm \mathbf{0 . 0 8}$ & $0.22 \pm 0.15$ \\
\hline MDAPE $[\%]$ & $\mathbf{1 . 2 1} \pm \mathbf{1 . 3 3}$ & $1.44 \pm 0.71$ & $\mathbf{0 . 3 2} \pm \mathbf{0 . 0 7}$ & $0.37 \pm 0.06$ \\
\hline WOBBLE $[\%]$ & $\mathbf{1 . 2 1} \pm \mathbf{0 . 5 6}$ & $2.34 \pm 0.95$ & $\mathbf{0 . 2 7} \pm \mathbf{0 . 1 0}$ & $0.50 \pm 0.06$ \\
\hline TV & $60.97 \pm 2.53$ & $\mathbf{4 . 3 0} \pm \mathbf{0 . 7 4}$ & $43.59 \pm 0.54$ & $\mathbf{2 . 3 5} \pm \mathbf{0 . 1 9}$ \\
\hline Q $[\mathrm{mg}]$ & $317.71 \pm 51.84$ & $\mathbf{3 0 8 . 1 2} \pm \mathbf{5 0 . 2 5}$ & $550.93 \pm 94.65$ & $\mathbf{5 5 0 . 8 9} \pm \mathbf{9 4 . 5 9}$ \\
\hline
\end{tabular}

Table 13: Mean values and standard deviations of the performance indexes obtained with the standard and the event-based PID controller for the setpoint response and disturbance rejection by considering all the patients of the dataset.

Other important indexes considered in this paper are the total variation (11) and the quantity of administered drug ( $[\mathrm{mg}])$. The obtained results are shown in Table 13.

A negative MDPE indicates that the controller tends to overdose, leading to BIS levels below the set-point, while a positive MDPE implies underdosing. In the set-point response, MDPE is lower for standard PID than for the event-based controller, but their values are in any case close to zero and this does not involve a significant difference from a clinical point of view. For the disturbance rejection task, MDPE is negative for PIDPlus and positive for the standard case, but also in this context the indexes are close to zero. The differences in the MDPE values can be attributed to the different tunings of the systems that can be more or less aggressive and therefore produce different values of PE.

A tighter control is expected from a system with a smaller MDAPE. This may reduce periods of excessive anesthesia or reduce risk of awareness. No significant differences occur in set-point response, where the MDAPE values are similar and stay within a range of $5 \%$, which is acceptable from a clinical standpoint. Also for disturbance rejection MDAPE is less than $5 \%$ but it is higher for PIDPlus than for the standard PID. The difference is due to the previously mentioned slower reaction of the event-based system, but this does not represent a problem because the settling time specifications are always fulfilled. The MDAPE different values also affect the WOBBLE analysis where there are some differences for disturbance rejection. The quantity of administered drug is similar for the two solutions with a slightly smaller value in set-point response for the event-based controller. Nevertheless, neither the MDAPE nor the WOBBLE allows the user to prefer the standard PID to the event-based PID controller, because the indexes have reasonable values in both cases. What makes the real difference between the two controllers is the total variation. For the standard PID controller its value can be also twenty times higher than for the event-based controller, 
mainly because of the effect of the derivative action on the measurement noise.

\section{Discussion}

Based on the obtained results the following considerations can be done:

- the event-based controller provides the required performance (fast transient and small overshoot) in the induction phase. Indeed, the performance is similar to that obtained with recently proposed control strategies like those based on model predictive control $[39,40]$ or on a state observer [42];

- also in the maintenance phase the event-based controller provides a satisfactory performance with limited BIS oscillations and undershoots. In the clinical practice the anesthesiologist has to manually compensate the disturbance with small propofol boluses. However, it might be difficult for him/her to determine the correct amount of drug to be administrated, especially in those cases where the BIS level suddenly changes due to the noxious stimulus;

- both the $I A E$ and $T V$ indexes have very similar values with and without noise and this proves that the influence of the noise is practically canceled without introducing a phase shift and therefore demonstrates the efficacy of the filtering property of the proposed event generator;

- the event-based solution can be preferred to a standard PID controller because, in spite of a negligible performance decrease detected with the performance indexes, the new event generator yields a much better control action. Indeed, less variations in the control variable are advantageous for the pump hardware and also for the patient, as a more regular infusion implies a more stable hemodynamic. The PI solution to eliminate the residual noise on the control action is not a very efficient solution because there is a significant decrement of the performance of the system with possibly dangerous undershoots;

- the control action resembles the manual infusion provided by the anesthesiologist, who can therefore clearly understand the behavior of the controller and better supervise the overall procedure;

- the new control architecture together with the determined optimal values of the parameters have demonstrated to provide the required robustness to inter- and intra-patient variability.

In conclusion, the proposed event-based control structure fulfills the clinical specifications by achieving a DoH slightly worse than that of a PID control, 
but with no residual noise and a piecewise constant control action beneficial for the patient, for the anesthesiologist, and for the pump.

\section{Conclusions}

The use of the event-based PID control methodology for the automatic dosing of propofol during general anesthesia has been developed in this paper in order to replicate the behavior of the anesthesiologist. Two different sets of parameters have been used for the induction and maintenance phases. The method has the clear advantage of providing a noise-free manipulated variable and this is paid by only a slight decrement of the performance with respect to PID control. The robustness to inter- and intra-patient variability has been demonstrated by applying a Monte Carlo method.

\section{References}

[1] A. Abdulla, P. Wen, and W. Xiang. The design and investigation of model based internal model control for the regulation of hypnosis. In Proceedings IEEE International Conference on Nano/Molecular Medicine and Engineering, pages 192-197, 2010.

[2] A. R. Absalom and G. N. Kenny. Closed-loop control of propofol anaesthesia using bispectral index: performance assessment in patients receiving computer controlled propofol and manually controlled remifentanil infusions for minor surgery. British Journal of Anaesthesia, 90:737-741, 2003.

[3] A. R. Absalom, R. De Keyser, and M. M. R. F. Struys. Closed loop anaesthesia: Are we getting close to finding the holy grail? Anesthesia Analgesia, 112:516-518, 2011.

[4] A. R. Absalom, N. Sutcliffe, and G. N. Kenny. Closed-loop control of anesthesia using bispectral index. Anesthesiology, 96:67-73, 2002.

[5] H. Araujo, B. Xiao, C. Liu, Y. Zhao, and H. K. Lam. Design of type1 and interval type-2 fuzzy PID control for anesthesia using genetic algorithms. Journal of Intelligent Learning Systems and Applications, 4:70-93, 2014.

[6] K. J. Åström. Analysis and design of nonlinear control systems. Springer, 2015.

[7] K. J. Åström and T. Hägglund. Advanced PID Control. ISA Press, Research Triangle Park, USA, 2006. 
[8] J. M. Bailey and W. M. Haddad. Drug dosing control in clinical pharmacology. IEEE Control Systems Magazine, 25(2):35-51, 2005.

[9] T. Blevins. PID advances in industrial control. In Proceedings IFAC Conference on Advances in PID Control, pages 23-28, Brescia, Italy, 2012 .

[10] T. Blevins, D. Chen, M. Nixon, and W. Wojsznis. Wireless Control Foundation: Continuous and Discrete Control for the Process Industry. ISA Press, 2015.

[11] T. Blevins, M. Nixon, and W. Wojsznis. PID control using wireless measurements. In Proceedings American Control Conference, pages 790-795, Portland, Oregon, 2014.

[12] M. T. Chan, B. C. Cheng, T. M. Lee, and T. Gin. Bis-guided anesthesia decreases postoperative delirium and cognitive decline. Journal of Neurosurgical Anesthesiology, 25:33-42, 2013.

[13] I. Constant and A. Rigouzzo. Which model for propofol TCI in children. Pediatric Anesthesia, 20:233-239, 2010.

[14] G. A. Dumont, A. Martinez, and J. M. Ansermino. Robust control of depth of anesthesia. International Journal of Adaptive Control and Signal Processing, 23:435-454, 2009.

[15] E. Elbetagi, T. Hegazy, and D. Grierson. Comparison among finve evolutionary-based optimization algorithms. Advanced Engineering Informatics, 19:43-53, 2005.

[16] M. S. Fadali and A. Visioli. Digital Control Engineering - Analysis and Design. Elsevier, 2012.

[17] W. M. Haddad, J. M. Bailey, T. Hayakawa, and N. Hovakimyan. Neural network adaptive output feedback control for intensive care unit sedation and intraoperative anesthesia. IEEE Transactions on Neural Networks, 18:1049-1066, 2007.

[18] J.-O. Hahna, G. A. Dumont, and J. M. Ansermino. Robust closed-loop control of hypnosis with propofol using $\mathrm{WAV}_{C N S}$ index as the controlled variable. Biomedical Signal Processing and Control, 7:517-524, 2012.

[19] L. Hemachandra. Monitoring Technologies in Acute Care Environments (J. M. Ehrenfeld and M. Cannesson eds.), chapter Target-controlled infusions, pages 281-287. Springer, 2014.

[20] C. M. Ionescu, R. De Keyser, B. C. Torrico, T. De Smet, M. M. R. F. Struys, and J. E. Normey-Rico. Robust predicitve control strategy 
applied for propofol dosing using BIS as a controlled variable during anesthesia. IEEE Transactions on Biomedical Engineering, 55(9):2161$2170,2008$.

[21] M. Janda, O. Simanski, J. Bajorat, B. Pohl, G. F. E. NoeldgeSchomburg, and R. Hofmockel. Clinical evaluation of a simultaneous closed-loop anaesthesia control system for depth of anaesthesia and neuromuscular blockade. Anesthesia, 66:1112-1120, 2011.

[22] G. N. C. Kenny and H. Mantzaridis. Closed-loop control of propofol anaesthesia. British Journal of Anaesthesia, 83:223-228, 1999.

[23] K. Leslie and T. G. Short. Low bispectral index values and death: the unresolved causality dilemma. Anesthesia and Analgesia, 113:660-663, 2011.

[24] M. L. Lindholm, S. Traff, F. Granath, S. D. Greenwald, A. Ekbom, C. Lennmarken, and R. H. Sandin. Mortality within 2 years after surgery in relation to low intraoperative bispectral index values and preexisting malignant disease. Anesthesia and Analgesia, 108(2):508$512,2009$.

[25] N. Liu, T. Chazot, A. Genty, A. Landais, A. Restoux, K. McGee, P. A. Laloe, B. Trillat, L. Barvais, and M. Fischler. Titration of propofol for anesthetic induction and maintenance guided by the bispectral index: closed-loop versus manual control. Anesthesiology, 104:686-695, 2006.

[26] N. Liu, T. Chazot, S. Hamada, A. Landais, N. Boichut, C. Dussaussoy, B. Trillat, L. Beydon, E. Samain, D. I. Sessler, and M. Fischler. Closed-loop coadministration of propofol and remifentanil guided by bispectral index: a randomized multicenter study. Anesthesia $\&$ Analgesia, 112:546-557, 2011.

[27] N. Liu, T. Chazot, B. Trillat, M. Michel-Cherqui, J. Y. Marandon, J.D. Law-Koune, B. Rives, and M. Fischler. Closed-loop control of consciousness during lung transplantation: an observational study. Journal of Cardiothoracic and Vascular Anesthesia, 22:611-615, 2008.

[28] N. Liu, E. M. Hafiani, and M. Le Guen. Monitoring Technologies in Acute Care Environments (J. M. Ehrenfeld and M. Cannesson eds.), chapter Closed-loop anesthesia based on neuromonitoring, pages 275279. Springer, 2014.

[29] M. Guinaldo Losada, F. Rodriguez Rubio, and S. Dormido Bencomo (editors). Asynchronous Control for Networked Systems. Springer, 2015 . 
[30] Emerson Process Management. Deltav v11 pid enhancements for wireless. Technical report, DeltaV white paper, 2013.

[31] V. Mani and N. S. Morton. Overview of total intravenous anesthesia in children. Pediatric Anesthesia, 20:211-222, 2010.

[32] A. Martinez. Robust control: PID vs. fractional control design, a case study. Master's thesis, Instituto Tecnologico de Estudios Superiores de Monterrey (MEX), 2000.

[33] C.F. Minto and T.W. Schnider. Contributions of PK/PD modeling to intravenous anesthesia. Clinical Pharmacology $\&$ Therapeutics, 84:2738, 2008.

[34] M. Miskowicz. Send-on-delta: An event-based data reporting strategy. Sensors, 6:49-63, 2006.

[35] M. Miskowicz. Event-Based Control and Signal Processing. CRC Press, 2015 .

[36] M. Mitchell. An Introduction to Genetic Algorithms. MIT press, Cambridge, USA, 1998.

[37] M. Morari and A. Gentilini. Challenges and opportunities in process control: Biomedical processes. AIChE J., 47(10):2140-2143, 2001.

[38] I. Nascu, A. Krieger, C. M. Ionescu, and E. Pistikopoulos. Advanced model-based control studies for the induction and maintenance of intravenous anesthesia. IEEE Transactions on Biomedical Engineering, 62:832-841, 2015.

[39] I. Nascu, R. Oberdieck, and E. N. Pistikopoulos. Explicit hybrid model predictive control strategies for intravenous anaesthesia. Computers and Chemical Engineering, 2017.

[40] I. Nascu and E. N. Pistikopoulos. Modeling, estimation and control of the anaesthesia process. Computers and Chemical Engineering, 2017.

[41] F. N. Nogueira, T. Mendonca, and P. Rocha. Controlling the depth of anesthesia by a novel positive control strategy. Computer Methods and Programs on Biomedicine, 114(3):e87-e97, 2014.

[42] F. N. Nogueira, T. Mendonca, and P. Rocha. Positive state observer for the automatic control of the depth of anesthesiaclinical results. Computer Methods and Programs on Biomedicine, 2017.

[43] F. Padula, C.Ionescu, N. Latronico, M. Paltenghi, A. Visioli, and G. Vivacqua. Optimized PID control of depth of hypnosis in anesthesia. Computer Methods and Programs in Biomedicine, 144:21-35, 2017. 
[44] F. Padula, C. Ionescu, N. Latronico, M. Paltenghi, A. Visioli, and G. Vivacqua. Inversion-based propofol dosing for intravenous induction of hypnosis. Communications in Nonlinear Science and Numerical Simulation, 39:481-494, 2016.

[45] G. D. Puri, B. Kumar, and J. Aveek. Closed-loop anaesthesia delievery system (CLADS) using bispectral index; a performance assessment study. Anaesthesia and Intensive Care, 35:357-362, 2007.

[46] C. Rosow and PJ. Manberg. Bispectral index monitoring. Anesthesiol Clin North America, 19(4):947-51, 2001.

[47] J. Sanchez, M. A. Guarnes, and S. Dormido. On the application of different event-based sampling strategies to the control of a simple industrial process. Sensors, 9:6795-6818, 2009.

[48] J. Sanchez, A. Visioli, and S. Dormido. PID Control in the Third Millennium (R. Vilanova and A. Visioli (eds.)), chapter Event-based PID control, pages 495-526. Springer, 2012.

[49] T. W. Schnider, C. F. Minto, P. L. Gambus, C. Andresen, D. B. Goodale, S. L. Shafer, and E. J. Youngs. The influence of method of administration and covariates on the pharmacokinetics of propofol in adult volunteers. Anesthesiology, 88:1170-1182, 1998.

[50] T. W. Schnider, C. F. Minto, S. L. Shafer, P. L. Gambus, C. Andresen, D. B. Goodale, and E. J. Youngs. The influence of age on propofol pharmacodynamics. Anesthesiology, 90:1502-1516, 1999.

[51] K. Soltesz. On Automation in Anesthesia. PhD thesis, Lund University (S), 2013.

[52] K. Soltesz, J.-O. Hahna, T. Hagglund, G. A. Dumont, and J. M. Ansermino. Individualized closed-loop control of propofol anesthesia: a preliminary study. Biomedical Signal Processing and Control, 8:500-508, 2013.

[53] J. Song, A. K. Mok, D. Chen, M. Nixon, T. Blevins, and W. Wojsznis. Improving PID control with unreliable communications. In Proceedings ISA EXPO, Houston, Texas, 2006.

[54] M. M. R. F. Struys, T. De Smet, S. Greenwald, A. R. Absalom, S. Binge, and E. P. Mortier. Performance evaluation of two published closed-loop control systems using bispectral index monitoring: a simulation study. Anesthesiology, 95(1):6-17, 2004.

[55] M. M. R. F. Struys, H. Vereecke, A. Moerman, E. W. Jensen, D. Verhaeghen, and N. De Neve. Ability of the bispectral index, autoregressive 
modelling with exogenous input-derived auditory evoked potentials, and predicted propofol concentrations to measure patient responsiveness during anesthesia with propofol and remifentanil. Anesthesiology, 99:802-812, 2003.

[56] A. L. G. Vanluchene, H. Vereecke, O. Thas, E. P. Mortier, S. L. Shafer, and M. M. R. F. Struys. Spectral entropy as an electroencephalographic measure of anesthetic drug effect. a comparison with bispectral index and processed midlatency auditory evoked response. Anesthesiology, 101:34-42, 2004.

[57] X. Y. Yang, Z. B. Zhou, L. Yang, X. Zhou, L. J. Niu, and X. Feng. Hemodynamic responses during induction: comparison of marsh and schnider pharmacokinetic models. International Journal of Clinical Pharmacology and Therapeutics, 53:32-40, 2015.

[58] S. Yelneedi, L. Samavedham, and G. P. Rangaiah. Advanced control strategies for the regulation of hypnosys with propofol. Industrial and Engineering Chemistry Research, 48:3880-3897, 2009.

[59] T. Zikov, S. Bibian, G. A. Dumont, M. Huzmezan, and C. R. Ries. Quantifying cortical activity during general anesthesia using wavelet analysis. IEEE Transactions on Biomedical Engineering, 53:617-632, 2006. 

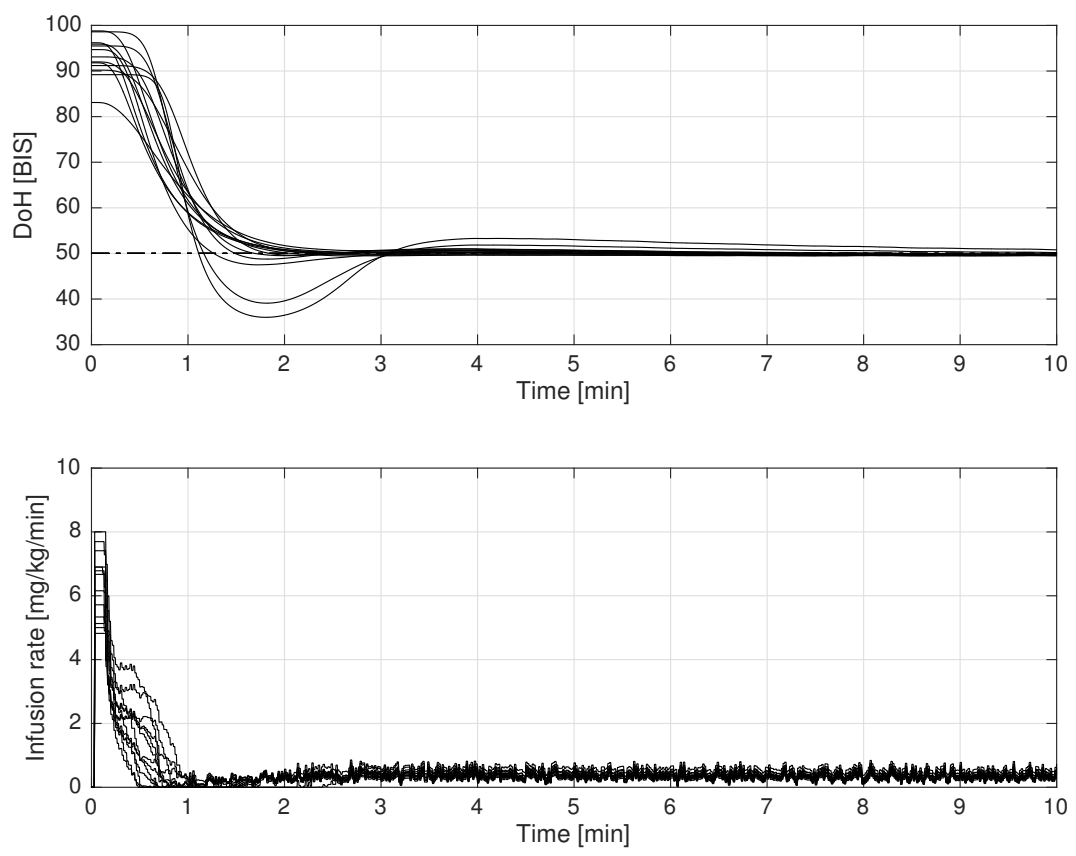

(a) Set-point response
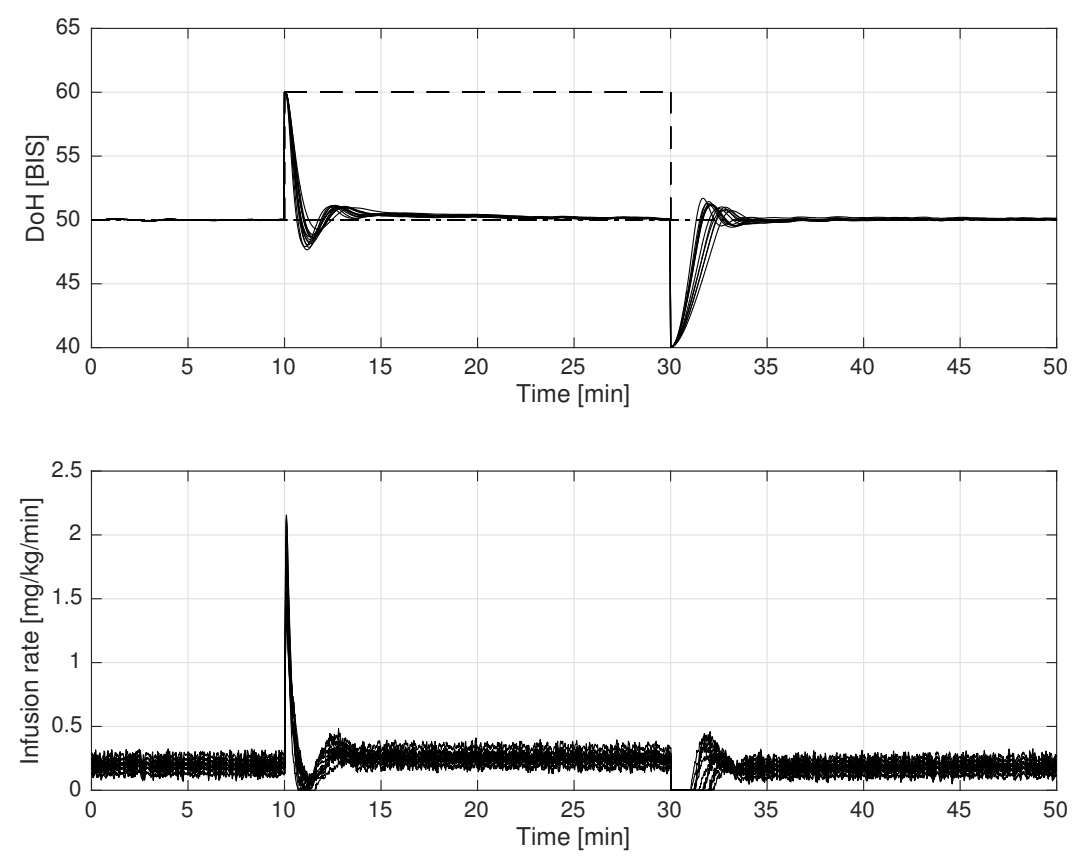

(b) Disturbance rejection

Figure 19: Set-point response and disturbance rejection for all the patients with a standard PID controller. 

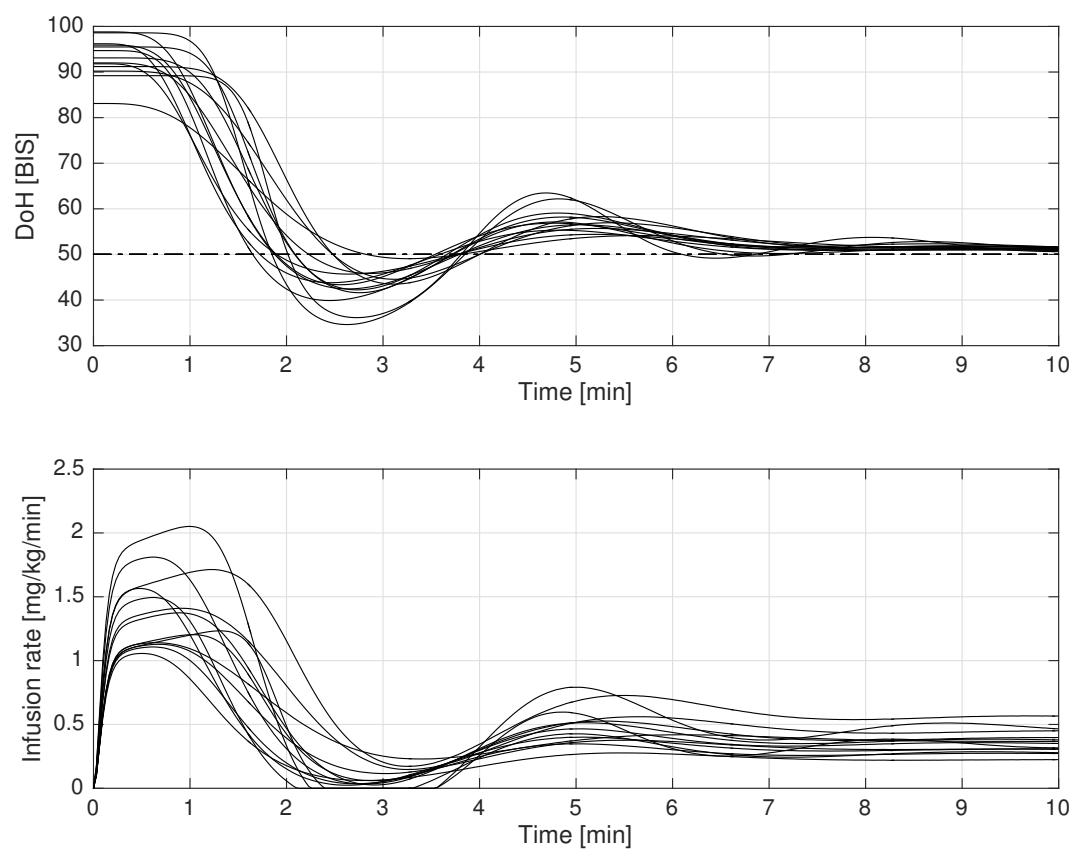

(a) Set-point response
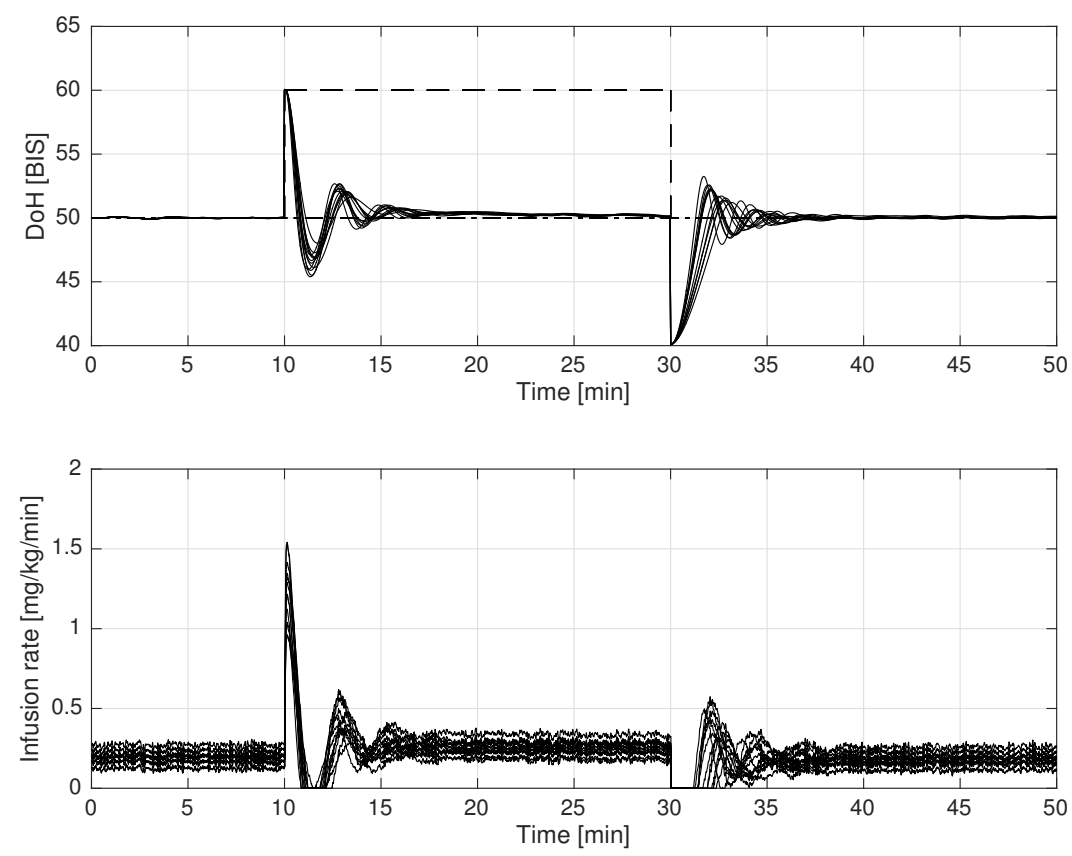

(b) Disturbance rejection

Figure 20: Set-point response and disturbance rejection for all the patients with a standard PI controller. 

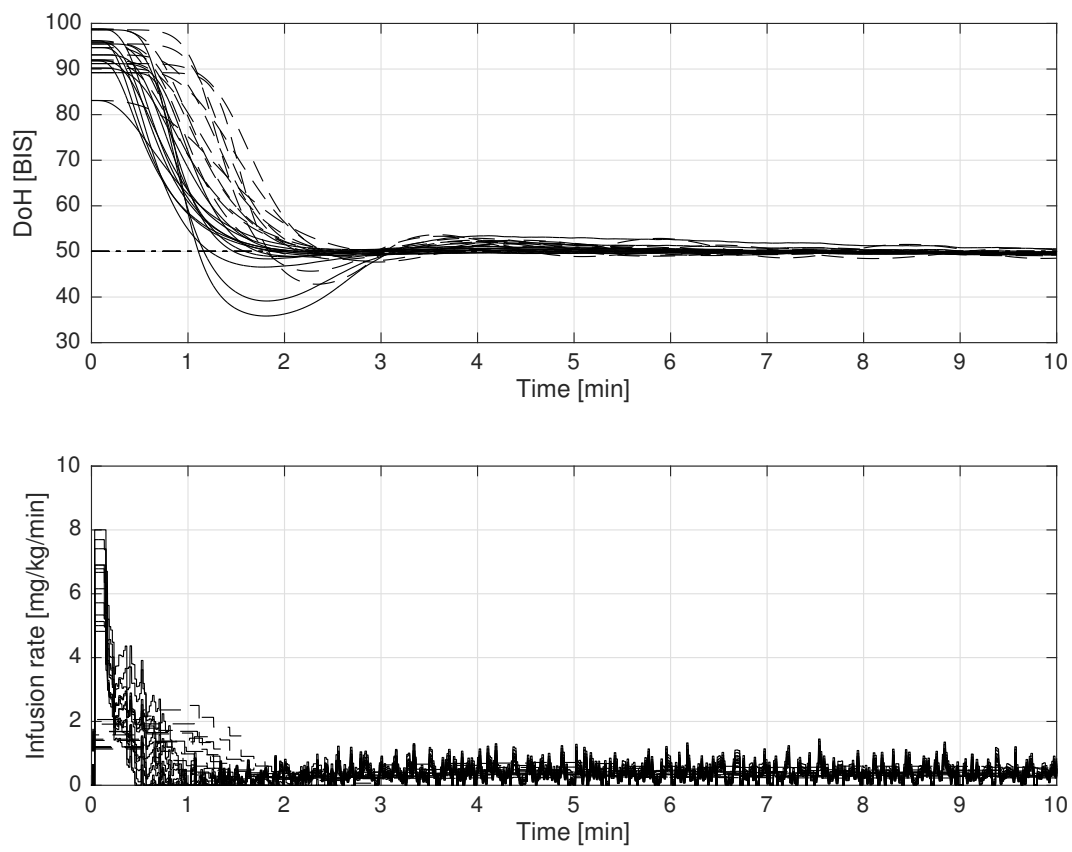

(a) Set-point response
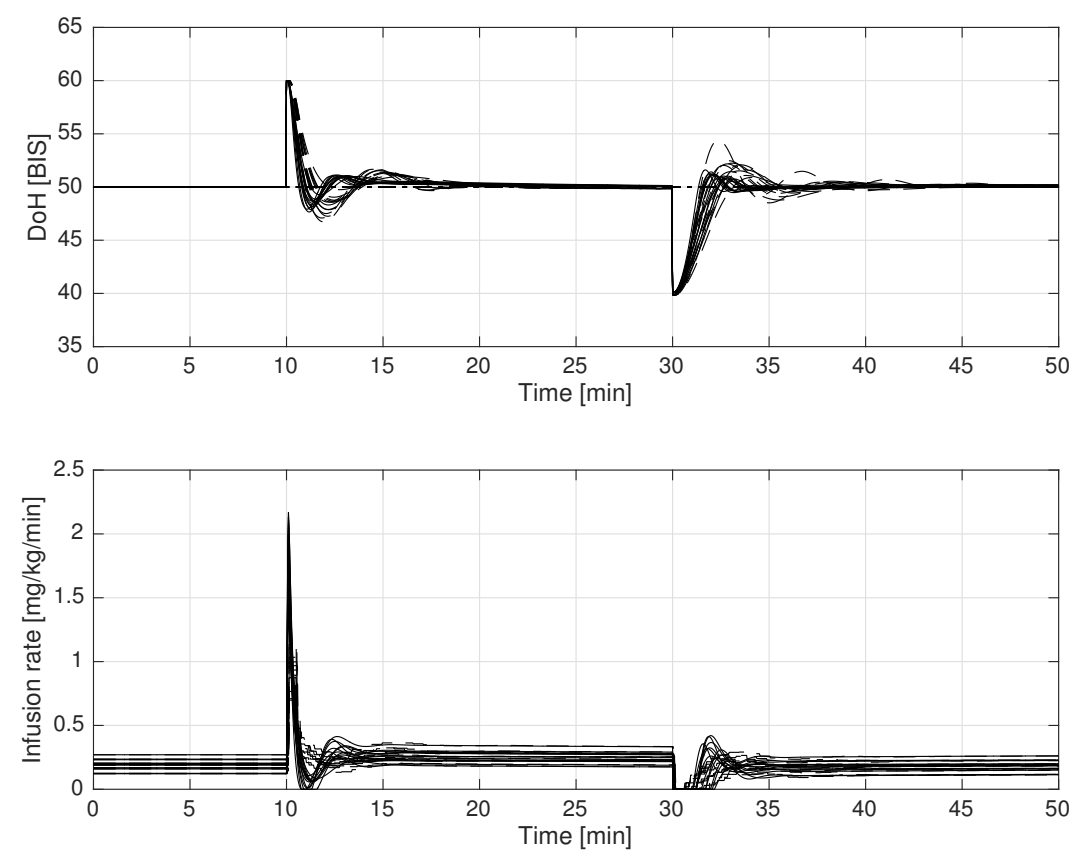

(b) Disturbance rejection

Figure 21: Set-point response and disturbance rejection for all the patients with a standard PID controller (solid lines) and with an event based PID system (dashed lines). 